Acta Math. Acad. Sci. Hung. 8 (1957) pp. 337-374

\title{
ON STOCHASTIC SET FUNCTIONS. II
}

\author{
András Prékopa (Budapest) \\ (Presented by A. Rényi) \\ Institution for Applied Mathematics of the Hungarian Academy of Sciences
}

Received: July 26, 1957

\section{Introduction}

The present paper contains an outline of the stochastic integral which can be defined relative to a completely additive stochastic set function $\xi(A)(A \in \mathcal{S})$.

Many types of stochastic integrals are known in the probability theory. Historically the first one is due to N. Wiener [21]. This was generalized by K. ITô by omitting the condition that the integrand is a constant function [10] and in another direction by considering a multidimensional Wiener process instead of the simple one [11]. An important step in the development was the integral representation of stochastic processes with independent increments. This result is essentially due to P. LÉvi [16] but a rigorous and complete treatment was given by K. ITô [9] (see also [12]). A stochastic Stieltjes integral with respect to a stochastic integral process with independent increments is used in [12]. The stochastic integral occurring in the theory of stationary processes was introduced by A. N. Kolmogorov [14] [15] and discussed from a general point of view by A. OBukhov [17]. These integrals were later generalized by J. L. Doob [5]. Another generalization is due to S. Bochner [1] for the case of an abstract space and for an additive random set function. Recently V. FABIAN [6] has defined a stochastic integral with a non-negative stochastic measure.

The generality of our integral introduced in $\S 1$ of Chapter I is contained in the following: The space where we integrate is abstract and we do not suppose at all the existence of any moments. This makes our integral of new type since in the above-mentioned stochastic integrals, except that of V. FABIAN who uses the non-negativity of the random measure, the existence of at least one moment of order $p \geq 1$ is always supposed. The speciality of the integral (1.3) is that the stochastic set function $\xi(A)(A \in \mathcal{S})$ has the following property: to disjoint sets $A_{1}, \ldots, A_{n}$ there correspond independent random variables. But exactly this property makes possible the proof of the existence and many properties of our integral.

We introduce a weaker and a stronger form of our integral. That one introduced by Definition 4 , the $u$-integral is a generalization of the Radon integral and reduces to the 
latter if the random variables $\xi(A)(A \in \mathcal{S})$ are constants with probability 1 . Most of the properties which are true in case of the Radon integral remain true in case of the $u$-integral too.

In the formulation of the theorems we did not take into consideration those trivial generalizations in which the difference from the original theorem is the neglect of some 0 -sets. This generalizations can be formulated without any difficulty.

The whole theory of our integral is based on the following property of completely additive stochastic set functions: the set of the distribution functions $\{F(x, A), A \in \mathcal{S}\}$ is (conditionally) compact in the space of the one-dimensional distribution functions. This theorem is proved in [18] (Chapter III, Theorem, 3.4).

To the practical applications we shall return later.

I express my sincere thanks to Professors Á. Császár, B. Sz.-Nagy and A. Rényi for their valuable remarks made in the preparation of this paper.

\section{Definitions and notations}

We keep all the notions and notations introduced in [18] (pp. 217-218). In the whole paper $H$ denotes the basic space where we integrate, $\mathcal{S}$ a $\sigma$-ring of some subsets of $H$ and $\xi(A)(A \in \mathcal{S})$ a completely additive set function with respect to which we integrate. The variable element of $H$ will be denoted by $h$. Only in Chapter III will be considered integrals with respect to more than one set functions, and in Chapter IV in some statements we replace the $\sigma$-ring $\mathcal{S}$ by a ring $\mathcal{R}$.

If we say that $\varphi(h)$ is integrable, we mean that it is integrable with respect to the completely additive set function $\xi(A)(A \in \mathcal{S})$. The terminology " $\varphi(h)$ is integrable" includes the measurability of the function.

We have to introduce only one new notion which did not take place in [18]. This is the following: a set $X \in \mathcal{S}$ is said to be a 0 -set with respect to the completely additive set function $\xi(A)(A \in \mathcal{S})$ if for every $Y \in X \mathcal{S}$ we have $\xi(Y)=0$.

\section{DEFINITION AND EXISTENCE OF THE INTEGRAL}

\section{$\S$ 1. Definitions}

In order to simplify our expressions we introduce the following definitions:

DEFINITION 1. A sequence of real numbers $\left\{y_{k}\right\}$ which is infinite in two directions will be called a dividing point sequence if $y_{k}<y_{k+1}(k=0, \pm 1, \pm 2, \ldots), \sup _{k}\left(y_{k+1}-y_{k}\right)<\infty$, $\lim _{k \rightarrow \infty} y_{k}=\infty$ and $\lim _{k \rightarrow-\infty} y_{k}=-\infty$. A sequence of dividing point sequences $\left\{y_{k}^{(n)}\right\}$ will be called a dividing point double sequence. 
DEFINITION 2. A dividing point double sequence $\left\{y_{k}^{(n)}\right\}$ will be called infinitely fine if

$$
\lim _{n \rightarrow \infty} \sup _{k}\left(y_{k+1}^{(n)}-y_{k}^{(n)}\right)=0 \text {. }
$$

Let $\varphi(h)$ be a real function defined on the elements of $H$ and measurable with regard to the $\sigma$-ring $\mathcal{S}$. Let furthermore $\left\{y_{k}\right\}$ be a dividing point sequence and consider the series of independent random variables

$$
\sum_{k=-\infty}^{\infty} y_{k} \xi\left(A H_{k}\right)
$$

where $A \in \mathcal{S}, H_{k}=\left\{h: y_{k} \leq \varphi(h)<y_{k+1}\right\}$. The sets $H_{k}$ are disjoint and their union equals $H$.

The system of sets $\left\{H_{k}\right)$ will be called the subdivision corresponding to the function $\varphi(h)$ and the dividing point sequence $\left\{y_{k}\right\}$. Now we formulate the definition of the integral.

Definition 3. Let $\varphi(h)(h \in H)$ be a measurable function and suppose that there exists a $\delta>0$ such that for every dividing point sequence $\left\{y_{k}\right\}$ with $\sup _{k}\left(y_{k+1}-y_{k}\right) \leq \delta$ the series (1.1) converges with probability 1 regardless of the order of summation. Let furthermore $\left\{y_{k}^{(n)}\right\}$ be an infinitely fine dividing point double sequence and put

$$
\eta_{n}(A)=\sum_{k=-\infty}^{\infty} y_{k}^{(n)} \xi\left(A H_{k}^{(n)}\right) .
$$

If the sequence (1.2) converges stochastically to a limiting random variable $\eta(A)$, then $\varphi(h)$ will be called integrable over the set $A$. The random variable $\eta(A)$, which is obviously independent of the special choice of $\left\{y_{k}^{(n)}\right\}$, will be called the integral of $\varphi(h)$ over the set $A$ and will be denoted by

$$
\int_{A} \varphi(h) \xi(\mathrm{d} A)
$$

We shall prove (Theorem 1.3) that if (1.1) converges for every sufficiently fine dividing point sequence, then the integral (1.3) exists. The integral introduced by Definition 3 has, however, some undesirable properties. First of all we mention that the classical Radon integral is not a special case of this. For instance, the function $\psi(h)$ defined by $(2.19)$ is not integrable in the sense of Radon with respect to the generalized measure (2.18), but is integrable in the sense of Definition 3. This implies other unpleasant properties as follows: if $\varphi(h)$ and $\psi(h)$ are measurable functions, $\psi(h)$ is integrable over the set $A$ and $|\varphi(h)| \leq|\psi(h)|$, then $\varphi(h)$ is not necessarily integrable over $A$. The same holds for the sum of two integrable functions $\varphi_{1}(h), \varphi_{2}(h)$. Both cases are illustrated by examples in Chapter II, $\S 3$.

In order to avoid the above-mentioned anomalies we introduce another integrals supposing more on the function $\varphi(h)$. 
DeFinition 4. A measurable function $\varphi(h)$ will be called unconditionally integrable (u-integrable) over the set $A \in \mathcal{S}$ if it is integrable in the sense of Definition 3 over all measurable subsets of $A$.

Now, the $u$-integral is a generalization of the Radon integral. In fact, if the stochastic set function $\xi(A)(A \in \mathcal{S})$ reduces to a number-valued completely additive set function $\mu(A)(A \in \mathcal{S})$, then, according to the decomposition theorem of HAHN, every set $A \in \mathcal{S}$ is the sum of two disjoint measurable sets $A_{1}, A_{2}$ with the property that

$$
\begin{array}{ll}
\mu(B) \geq 0 \quad \text { for } & B \in A_{1} \mathcal{S}, \\
\mu(B) \leq 0 \quad \text { for } & B \in A_{2} \mathcal{S} .
\end{array}
$$

The $u$-integrability of $\varphi(h)$ implies the existence of the Lebesgue integrals

$$
\int_{A_{1}} \varphi(h) \mu(\mathrm{d} A), \quad \int_{A_{2}} \varphi(h)(-\mu(\mathrm{d} A)),
$$

hence our assertion follows.

It will be shown that the $u$-integral has analogous properties to the Radon integral.

We anticipate a special case of Theorem 2.7. If $\varphi(h)$ is measurable and $|\varphi(h)|$ is $u$ integrable over $A \in \mathcal{S}$, then the same holds for $\varphi(h)$. The conversion is also true. In fact, if $\varphi(h)$ is measurable and $u$-integrable, then the positive and negative parts of $\varphi(h)$ are $u$-integrable, hence, using Theorem 2.2 , the statement follows.

\section{$\S 2$. Two auxiliary theorems}

In the paper [18] we have introduced the following set function:

$$
W(T, A)=\operatorname{Var}_{\alpha}(A) \quad(A \in \mathcal{S}),
$$

where

$$
\alpha(T, B)=\sup _{|t| \leq T}|1-f(t, B)| \quad(B \in \mathcal{S})
$$

and $T$ is an arbitrary but fixed positive number. In order to avoid superfluous complications in the formulae of the present paper we extend the definition of $W(T, A)(A \in \mathcal{S})$ by writing

$$
W(-T, A)=W(T, A),
$$

$W(0, A)=0$. Thus for a fixed $A W(I, A)$ is an even function defined on the whole real axis. It is shown in [18] (cf. the proof of Theorem 3.2) that the set function (1.4) is a finite (consequently also bounded) measure on the $\sigma$-ring $\mathcal{S}$. In the sequel this measure will have a fundamental role.

The first theorem refers to this and to a closely related set function. 
Theorem 1.1. For every set $A \in \mathcal{S}$

$$
\lim _{T \rightarrow 0} W(T, A)=0
$$

i.e. $W(T, A)$ is continuous at $T=0(W(0, A)$ is obviously equal to 0$)$. It is true furthermore that for every set $A \in \mathcal{S}$ the set function

$$
\operatorname{Var}_{\mu(a)}(A) \quad(\mu(a, B)=\mathbf{P}(|\xi(B)|>a), B \in \mathcal{S})
$$

depending on the number a is continuous at $a=\infty$, i.e.

$$
\lim _{a \rightarrow \infty} \operatorname{Var}_{\mu(a)}(A)=0
$$

Proof. Let $B_{1}, \ldots, B_{r}$ be a system of disjoint sets of the $\sigma$-algebra $A \mathcal{S}$. According to Theorem 3.4 of [18] for every $\varepsilon>0$ there can be found a $\delta>0$ such that

$$
-\log |f(t, C)| \leq \varepsilon, \quad|\arg f(t, C)| \leq \varepsilon,^{1}
$$

provided that $C \in \mathcal{S}$ and $|t| \leq \delta$. Let us divide the system $B_{1}, \ldots, B_{r}$ into two groups accordingly as $\arg f\left(t, B_{k}\right)>0$ or $\arg f\left(t, B_{k}\right) \leq 0$. By (1.9) we have (if $\varepsilon<\frac{2 \pi}{3}$ )

$$
\begin{gathered}
\sum_{k}^{\prime} \arg f\left(t, B_{k}\right)=\arg f\left(t, B^{\prime}\right) \leq \varepsilon, \\
-\sum_{k}^{\prime \prime} \arg f\left(t, B_{k}\right)=-\arg f\left(t, B^{\prime \prime}\right) \leq \varepsilon,
\end{gathered}
$$

where the summations $\sum^{\prime}$ and $\sum^{\prime \prime}$ refer to the subscripts of the sets belonging to the first and second groups, the sets $B^{\prime}$ and $B^{\prime \prime}$ denote the unions of the corresponding sets, respectively. (1.10) implies that

$$
\sum_{k=1}^{r}\left|\arg f\left(t, B_{k}\right)\right| \leq 2 \varepsilon
$$

if $|t| \leq \delta$. On the other hand, by (1.9) we have also

$$
-\log |f(t, B)| \leq \varepsilon \quad\left(B=B^{\prime}+B^{\prime \prime}\right)
$$

if $|t| \leq \delta$. Using (1.11) and (1.12) we get

$$
\begin{aligned}
\sum_{k=1}^{r} & \left|1-f\left(t, B_{k}\right)\right|=\sum_{k=1}^{r}\left|1-e^{i \arg f\left(t, B_{k}\right)}\right| f\left(t, B_{k}\right)|| \\
\leq & \sum_{k=1}^{r} \mid 1-e^{i \arg f\left(t, B_{k}\right) \mid}+\sum_{k=1}^{r}\left(1-\left|f\left(t, B_{k}\right)\right|\right) \\
\leq & \sum_{k=1}^{r}\left|\arg f\left(t, B_{k}\right)\right|-\sum_{k=1}^{r} \log \left|f\left(t, B_{k}\right)\right| \leq 2 \varepsilon-\log |f(t, B)| \leq 3 \varepsilon .
\end{aligned}
$$

\footnotetext{
${ }^{1}$ Under $\arg z$ we understand the main value of this function: $-\pi<\arg z \leq \pi$.
} 
Applying the inequality

$$
\frac{1}{2 a} \int_{-a}^{a}|1-f(t)| \mathrm{d} t \geq \frac{1}{10} \int_{|x|>\frac{1}{a}} \mathrm{~d} F(x),
$$

where $F(x)$ is an arbitrary distribution function, $f(t)$ is the corresponding characteristic function and $a$ is a positive number, we conclude

$$
\sum_{k=1}^{r} \mathbf{P}\left(\left|\xi\left(B_{k}\right)\right|>\frac{1}{a}\right) \leq \frac{10}{2 a} \sum_{k=1}^{r} \int_{-a}^{a}\left|1-f\left(t, B_{k}\right)\right| \mathrm{d} t \geq 30 \varepsilon
$$

whenever $\frac{1}{a} \geq \frac{1}{\delta}$. Thus the second assertion of our theorem is proved.

Our first assertion follows from the inequality

$$
\begin{aligned}
& \left|1-f\left(t, B_{k}\right)\right|=\left|\int_{-\infty}^{\infty}\left(1-e^{i t x}\right) \mathrm{d} F\left(x, B_{k}\right)\right| \\
& \quad \leq|t|\left|\int_{|x| \leq a} x \mathrm{~d} F\left(x, B_{k}\right)\right|+\frac{t^{2}}{2} \int_{|x| \leq a} x^{2} \mathrm{~d} F\left(x, B_{k}\right)+2 \mathbf{P}\left(\left|\xi\left(B_{k}\right)\right|>a\right),
\end{aligned}
$$

where $a$ is a positive number and from Theorem 3.8 of [18]. This completes the proof.

The following theorem will be needed first of all in the proof of the existence of the integral (1.3).

THEOREM 1.2. Let $C_{k}^{(n)}(k, n=1,2, \ldots)$ be a system of sets of the $\sigma$-ring $\mathcal{S}$ for which $C_{i}^{(n)} C_{k}^{(n)}=0$ if $i \neq k(n=1,2, \ldots)$ and $\delta_{k}^{(n)}(k, n=1,2, \ldots)$ a double sequence of real numbers for which $\lim _{n \rightarrow \infty} \sup _{k}\left|\delta_{k}^{(n)}\right|=0$. In this case

$$
\sum_{k} \delta_{k}^{(n)} \xi\left(C_{k}^{(n)}\right) \Rightarrow 0
$$

if $n \rightarrow \infty$.

Proof. For a fixed $n$ the series (1.17) converges with probability 1 regardless of the order of summation. In fact, this holds for the series

$$
\sum_{k} \xi\left(C_{k}^{(n)}\right)
$$

and the set of numbers $\left\{\delta_{k}^{(n)}\right\}$ is bounded, hence an easy argument referring to the three series theorem of Kolmogorov shows the truth of the preceding assertion. 
Using an inequality of the type (1.16) we obtain

$$
\begin{aligned}
\left|1-\prod_{k} f\left(t \delta_{k}^{(n)}, C_{k}^{(n)}\right)\right| \leq & \sum_{k}\left|1-f\left(t \delta_{k}^{(n)}, C_{k}^{(n)}\right)\right| \\
\leq & |t| \sum_{k}\left|\delta_{k}^{(n)}\right|\left|\int_{|x| \leq a} x \mathrm{~d} F\left(x, C_{k}^{(n)}\right)\right| \\
& +\frac{t^{2}}{2} \sum_{k}\left(\delta_{k}^{(n)}\right)^{2} \int_{|x| \leq a} x^{2} \mathrm{~d} F\left(x, C_{k}^{(n)}\right)+2 \mathbf{P}\left(\left|\xi\left(C_{k}^{(n)}\right)\right|>a\right),
\end{aligned}
$$

where $a$ is a positive number. By Theorem 3.8 of [18] there is a number $K_{a}>0$ such that

$$
\begin{gathered}
\sum_{k}\left|\int_{|x| \leq a} x \mathrm{~d} F\left(x, C_{k}^{(n)}\right)\right| \leq K_{a}, \\
\sum_{k} \int_{|x| \leq a} x^{2} \mathrm{~d} F\left(x, C_{k}^{(n)}\right) \leq K_{a} .
\end{gathered}
$$

We may suppose that $K_{a} \geq 1$. First, using Theorem 1.1, we choose $a$ so large that the third member on the right-hand side of (1.18) is smaller than $\varepsilon$ (we suppose that $\varepsilon \leq 1$ ). Next we choose $n$ so that $\left|\delta_{k}^{(n)}\right| \leq \frac{\varepsilon}{K_{a}} \leq 1(k=1,2, \ldots)$. In this case the sum on the right-hand side of (1.18) is at most $\varepsilon\left(|t|+t^{2} / 2+1\right)$. Thus our theorem is proved.

\section{$\S 3$. The existence of the integral}

Now we return to our original problem, the discussion of the existence of the integral defined in $\S 1$. The main theorem regarding this matter is the following:

THEOREM 1.3. Let $\varphi(h)$ be a real function measurable with regard to the $\sigma$-ring $\mathcal{S}$ and $A \in \mathcal{S}$ a set. Suppose that there exists a $\delta>0$ such that the series (1.1) converges with probability 1 regardless of the order of summation whenever the dividing point sequence $\left\{y_{k}\right\}$ has the property $\sup _{k}\left(y_{k+1}-y_{k}\right) \leq \delta$. In this case the integral (1.3) exists. In particular, every measurable bounded function is integrable.

Proof. Let $\left\{y_{k}^{(n)}\right\}$ be an infinitely fine dividing point double sequence. Let us unite the sequences $\left\{y_{k}^{(n)}\right\},\left\{y_{k}^{(m)}\right\}$ and denote the new dividing point sequence by $\left\{z_{k}\right\} \cdot{ }^{2}$ If we introduce the notation

$$
L_{j}=\left\{h: z_{j} \leq \varphi(h)<z_{j+1}\right\},
$$

\footnotetext{
${ }^{2}$ For the sake of brevity we do not indicate that it depends on $n$ and $m$.
} 
then

$$
\begin{gathered}
\sum_{k} y_{k}^{(n)} \xi\left(A H_{k}^{(n)}\right)-\sum_{k} y_{k}^{(m)} \xi\left(A H_{k}^{(m)}\right) \\
=\sum_{k} \sum_{j: L_{j} \subseteq H_{k}^{(n)}}\left(y_{k}^{(n)}-z_{j}\right) \xi\left(A L_{j}\right)-\sum_{k} \sum_{j: L_{j} \subseteq H_{k}^{(m)}}\left(y_{k}^{(m)}-z_{j}\right) \xi\left(A L_{j}\right) .
\end{gathered}
$$

In the sums on the right-hand side the set of the numbers $y_{k}^{(n)}-z_{j}, y_{k}^{(m)}-z_{j}$ is bounded and if $\delta_{k j}^{(n)}=y_{k}^{(n)}-z_{j}$, then the properties of the double sequence $\left\{y_{k}^{(n)}\right\}$ imply that

$$
\lim _{n \rightarrow \infty} \sup _{k} \max _{j: L_{j} \subseteq H_{k}^{(n)}}\left|\delta_{k j}^{(n)}\right|=0 .
$$

Applying Theorem 1.2 it follows that the sequence of random variables in (1.19) tends stochastically to 0 as $n, m \rightarrow \infty$.

Thus the Cauchy's convergence criterion holds, hence there is a random variable to which the first member on the left-hand side of (1.19) converges stochastically. As it can be seen by a well-known argument, this limit is independent of the special choice of the double sequence $\left\{y_{k}^{(n)}\right\}$. Hence our theorem is proved.

REMARK. It is not difficult to see that if the series (1.1) converges for sufficiently fine dividing point sequences, then the same holds for any dividing point sequence. This and other statements occurring in our discussion follow from the fact that if the series of independent random variables

$$
\sum_{k=1}^{\infty} \xi_{k}
$$

converges with probability 1 regardless of the order of summation, then the same holds for

$$
\sum_{k=1}^{\infty} c_{k} \xi_{k},
$$

where $c_{k}$ is a bounded sequence of real numbers. ${ }^{3}$

Now we deduce an inequality playing an important role in the proofs of the present paper.

THEOREM 1.4. Let $\varphi(h)$ be a measurable function for which $|\varphi(h)| \leq K<\infty$. Then, denoting by $g(t, A)$ the characteristic function of the integral of $\varphi(h)$ over the set $A(A \in$ $\mathcal{S})$, we have

$$
|1-g(t, A)| \leq W(t K, A) .
$$

\footnotetext{
${ }^{3}$ This statement is a simple consequence of the three series theorem of KoLmogorov.
} 
Proof. Let $\left\{y_{k}^{(n)}\right\}$ be an infinitely fine dividing point double sequence and $\left\{H_{k}^{(n)}\right\}$ the corresponding sequence of subdivisions determined by $\varphi(h)$. If $g_{n}(t, A)$ denotes the characteristic function of the random variable

$$
\sum_{k} y_{k}^{(n)} \xi\left(A H_{k}^{(n)}\right)
$$

then

$$
\begin{gathered}
\left|1-g_{n}(t, A)\right| \leq \sum_{k}\left|1-f\left(t y_{k}^{(n)}, A H_{k}^{(n)}\right)\right| \\
\leq \sum_{k} W\left(t y_{k}^{(n)}, A H_{k}^{(n)}\right) \leq \sum_{k} W\left(t K, A H_{k}^{(n)}\right)=W(t K, A) .
\end{gathered}
$$

Taking the limit $n \rightarrow \infty$, we obtain our assertion.

\section{$\S 4$. A necessary condition for the existence of the integral (1.3)}

In this section we prove the following

Theorem 1.5. Let $\varphi(h)$ be a measurable function integrable over the set $A \in \mathcal{S}$ and $a_{n}, b_{n}(n=1,2, \ldots)$ a pair of sequences with the properties $a_{n+1} \leq a_{n}, b_{n+1} \geq b_{n}$ $(n=1,2, \ldots), \lim _{n \rightarrow \infty} a_{n}=-\infty, \lim _{n \rightarrow \infty} b_{n}=\infty$. In this case the sequence of random variables

$$
\eta_{n}=\int_{A_{n}} \varphi(h) \xi(\mathrm{d} A) \quad\left(A_{n}=\left\{h: h \in A, a_{n} \leq \varphi(h)<b_{n}\right\}\right)
$$

converges with probability 1 and

$$
\int_{A} \varphi(h) \xi(\mathrm{d} A)=\lim _{n \rightarrow \infty} \eta_{n}
$$

Proof. We may suppose that $a_{n}<0, b_{n}>0$,

$$
\sup _{n}\left(b_{n+1}-b_{n}\right)<\infty, \quad \sup _{n}\left(a_{n}-a_{n+1}\right)<\infty \quad(n=1,2, \ldots) .
$$

Let us unite these sequences into one dividing point sequence $\left\{y_{n}\right\}$ and consider the series

$$
\sum_{n} \int_{A H_{n}}\left(y_{n}-\varphi(h)\right) \xi(\mathrm{d} A),
$$

where $\left\{H_{n}\right\}$ is the subdivision determined by the sequence $\left\{y_{n}\right\}$ and the function $\varphi(h)$. We do not know yet that the series $(1.22)$ converges. Denoting by $f_{n}(t)$ the characteristic function of the $n$-th member of the series (1.22), it follows from Theorem 1.4 that

$$
\left|1-f_{n}(t)\right| \leq W\left(\delta t, A H_{n}\right)
$$


where $\delta=\sup _{n}\left(y_{n+1}-y_{n}\right)$. (1.23) implies

$$
\sum_{n}\left|1-f_{n}(t)\right| \leq W(\delta t, A) .
$$

As the terms of the series (1.22) are evidently independent, this series converges with probability 1 regardless of the order of summation (cf. [5], p. 115, Theorem 2.7). The sum (1.22) equals

$$
\sum_{n} y_{n} \xi\left(A H_{n}\right)-\sum_{n} \int_{A H_{n}} \varphi(h) \xi(\mathrm{d} A),^{4}
$$

hence, taking Theorem 1.3 into account, it follows that if $\varphi(h)$ is integrable over $A$, then the sequences of the type (1.20) converge with probability 1.

Now we are going to verify the relation (1.21). Let $\eta$ denote the limiting variable of the sequence $\eta_{n}$ and choose an infinitely fine dividing point double sequence $\left\{y_{n}^{(N)}\right\}$ with the property $\left\{y_{n}^{(N)}\right\} \subseteq\left\{y_{n}^{(N+1)}\right\}$ and $\left\{y_{n}^{(1)}\right\}=\left\{y_{n}\right\}$. If $\left\{H_{n}^{(N)}\right\}$ is the corresponding sequence of subdivisions determined by $\varphi(h)$, then, using the precedings, we have

$$
\sum_{n} \int_{A H_{n}^{(N)}} \varphi(h) \xi(\mathrm{d} A)=\eta
$$

for every $N$. Let $f_{n}^{(N)}(t)$ denote the characteristic function of the $n$-th term of the series

$$
\sum_{n} \int_{A H_{n}^{(N)}}\left(y_{n}^{(N)}-\varphi(h)\right) \xi(\mathrm{d} A)
$$

If $f^{(N)}(t)$ denotes the characteristic function of the sum (1.26), then by Theorem 1.4 we have

$$
\left|1-f^{N}(t)\right| \leq \sum_{n}\left|1-f_{n}^{(N)}(t)\right| \leq W\left(\delta^{(N)} t, A\right)
$$

where $\delta^{(N)}=\sup _{n}\left(y_{n+1}^{(N)}-y_{n}^{(N)}\right)$. By (1.27) and Theorem 1.1

$$
f^{(N)}(t) \Rightarrow 1 \quad \text { if } \quad N \rightarrow \infty
$$

(1.25) implies that the sequence (1.26) (which depends on $N$ ) converges stochastically to the random variable

$$
\int_{A} \varphi(h) \xi(\mathrm{d} A)-\eta
$$

Hence this is equal to 0 with probability 1.

\footnotetext{
${ }^{4}$ We have used here a trivial special case of Theorem 2.4
} 


\section{THE PROPERTIES OF THE INTEGRAL $\int_{A} \varphi(h) \xi(\mathrm{d} A)$}

\section{$\S 1$. Elementary properties of the integral}

It is only for the sake of the systematic treatment that we mention the following almost trivial theorems:

THEOREM 2.1. If $\varphi(h)$ is integrable over the set $A \in \mathcal{S}$, then the same holds also for $c \varphi(h)$ where $c$ is a real constant and

$$
\int_{A} c \varphi(h) \xi(\mathrm{d} A)=c \int_{A} \varphi(h) \xi(\mathrm{d} A) .
$$

Theorem 2.2. If $\varphi(h)$ is integrable over the sets $A_{1} \in \mathcal{S}, A_{2} \in \mathcal{S}$ where $A_{1} A_{2}=0$, then it is integrable also over $A_{1}+A_{2}$ and

$$
\int_{A_{1}+A_{2}} \varphi(h) \xi(\mathrm{d} A)=\int_{A_{1}} \varphi(h) \xi(\mathrm{d} A)+\int_{A_{2}} \varphi(h) \xi(\mathrm{d} A) .
$$

The following theorem requires a little more complicated argument than the corresponding theorem for Lebesgue integrals.

THEOREM 2.3. If $\varphi(h)$ is a measurable and bounded step function over the set $A \in \mathcal{S}$, i.e. there are disjoint sets $A_{1}, A_{2}, \ldots\left(\sum_{k=1}^{\infty} A_{k}=A\right)$ of $\mathcal{S}$ such that $\varphi(h)=\varphi_{k}$ if $h \in A_{k}$ and $\left|\varphi_{k}\right| \leq K(k=1,2, \ldots)$ where $K$ is a constant, then

$$
\int_{A} \varphi(h) \xi(\mathrm{d} A)=\sum_{k=1}^{\infty} \varphi_{k} \xi\left(A_{k}\right) .
$$

A similar but more general assertion follows from Theorem 2.4 for $u$-integrable functions taking on a countable number of different values.

\section{$\S 2$. The complete additiveness of the indefinite integral}

In this $\S$ our aim is to prove the following

Theorem 2.4. If the function $\varphi(h)$ is integrable over all sets $A \in \mathcal{S}$, then

$$
\eta(A)=\int_{A} \varphi(h) \xi(\mathrm{d} A)
$$

is a completely additive stochastic set function. ${ }^{5}$

\footnotetext{
${ }^{5}$ The definition of this notion is given in [18], p. 216.
} 
Proof. It is quite easy to argue that $\eta(A)$ is an additive set function. In fact, if the sets $A_{k} \in \mathcal{S}(k=1,2, \ldots, r)$ are disjoint, then the random variables $\eta\left(A_{k}\right)(k=1,2, \ldots, r)$ must be independent and Theorem 2.2 ensures the fulfilment of the remaining part of this assertion.

Now we prove that the set function $\eta$ is also completely additive. First we consider the case of a bounded function and suppose that $|\varphi(h)| \leq K$. According to Theorem 2.1 of [18] we have to verify the following criterion: if $B_{1}, B_{2}, \ldots$ is a non-increasing sequence of sets of $\mathcal{S}$ such that $\lim _{n \rightarrow \infty} B_{n}=0$, then

$$
\eta\left(B_{n}\right) \Rightarrow 0 \quad \text { if } \quad n \rightarrow \infty .
$$

Denoting by $f_{n}(t)$ the characteristic function of $\eta\left(B_{n}\right)$. Theorem 1.4 implies

$$
\left|1-f_{n}(t)\right| \leq W\left(K t, B_{n}\right) .
$$

As for every $T W(T, A)$ is a bounded measure on the $\sigma$-ring $\mathcal{S}$, it follows that

$$
\lim _{n \rightarrow \infty} W\left(K t, B_{n}\right)=0
$$

Our assertion follows from (2.6) and (2.7).

Now we consider the general case. Let us introduce the notation $\mathcal{S}_{N}=D_{N} \mathcal{S}$ where $D_{n}=\{h:-N \leq \varphi(h)<N\}$. We shall denote by $\mathcal{R}$ the ring of those sets $A$ which are of the form

$$
A=A_{i_{1}}+\cdots+A_{i_{r}} \quad \text { where } \quad A_{i_{k}} \in \mathcal{S}_{i_{k}} \quad(k=1, \ldots, r) .
$$

Our argument for the case of a bounded function shows that the set function $\eta$ is completely additive on $\mathcal{R}$. In fact, if $A_{1}, A_{2}, \ldots$ is a sequence of disjoint sets of $\mathcal{R}$ for which $A=\sum_{k=1}^{\infty} A_{k} \in \mathcal{R}$, then for some $N$ we have $A \in \mathcal{S}_{N}$. But since $A_{k} \subseteq A$ $(k=1,2, \ldots)$, we have also $A_{k} \in \mathcal{S}_{N}(k=1,2, \ldots)$. As $\varphi(h)$ is bounded on the $\sigma$-ring $\mathcal{S}_{N}$, it follows that

$$
\eta(A)=\sum_{k=1}^{\infty} \eta\left(A_{k}\right) .
$$

The following step is to prove that the set function $\eta$ can be extended to the smallest $\sigma$-ring $\mathcal{S}(\mathcal{R})$ containing $\mathcal{R}$ (cf. [18], p. 233). Obviously $\mathcal{S}(\mathcal{R})=\mathcal{S}$. Let $B_{1}, B_{2}, \ldots$ be an arbitrary sequence of disjoint sets of $\mathcal{R}$. We shall show that the series

$$
\sum_{k=1}^{\infty} \eta\left(B_{k}\right)
$$

converges with probability 1 regardless of the order of summation. By Theorem 3.2 of [18] this property ensures the possibility of the extension. Let

$$
\begin{gathered}
C_{N_{r} k_{s}^{(r)}}=\left(D_{N_{r}+1}-D_{N_{r}}\right) B_{k_{s}^{(r)}} \\
\left(N_{r}<N_{r+1}, k_{s}^{(r)}<k_{s+1}^{(r)} ; s=1,2, \ldots ; r=1,2, \ldots\right) .
\end{gathered}
$$


Since $|\varphi(h)| \leq N_{r}+1$ for $h \in C_{N_{r}}=\sum_{s=1}^{\infty} C_{N_{r} k_{s}^{(r)}}$, it follows that

$$
\eta\left(C_{N_{r}}\right)=\sum_{s=1}^{\infty} \eta\left(C_{N_{r} k_{s}^{(r)}}\right)
$$

and this series converges with probability 1 regardless of the order of summation. On the other hand, $\varphi(h)$ is integrable over the set $C=\sum_{r=1}^{\infty} C_{N_{r}}$, hence by Theorem 1.5 (choosing the sequences $a_{r}=-N_{r}-1, b_{r}=N_{r}+1$ )

$$
\eta(C)=\sum_{r=1}^{\infty} \eta\left(C_{N_{r}}\right) .
$$

(This series must converge also if we omit an arbitrary set of terms, hence it converges with probability 1 regardless of the order of summation. ${ }^{6}$ ) Comparing (2.9) and (2.10), it follows that the series

$$
\sum_{r=1}^{\infty} \sum_{s=1}^{\infty} \eta\left(C_{N_{r} k_{s}^{(r)}}\right)
$$

converges with probability 1 . Now we prove a

Lemma. Let $\xi_{i k}(i, k=1,2, \ldots)$ be independent random variables. Suppose that for every system of sequences $i_{r}, k_{s}^{(r)}(s=1,2, \ldots ; r=1,2, \ldots)$ with $i_{r}<i_{r+1}, k_{s}^{(r)}<k_{s+1}^{(r)}$ $(s=1,2, \ldots ; r=1,2, \ldots)$ the series

$$
\sum_{r=1}^{\infty} \sum_{s=1}^{\infty} \xi_{i_{r} k_{s}^{(r)}}
$$

converges with probability 1 . In this case the series

$$
\sum_{i=1}^{\infty} \sum_{k=1}^{\infty} \xi_{i k}
$$

converges with probability 1 regardless of the order of summation.

Proof. By condition there is a $\delta>0$ such that for every $t$ for which $|t| \leq \delta$, the infinite double product

$$
\prod_{r=1}^{\infty} \prod_{s=1}^{\infty} f_{i_{r} k_{s}^{(r)}}(t)
$$

converges where $f_{i k}(t)$ is the characteristic function of the random variable $\xi_{i k}$.

Let us fix the number $t(|t| \leq \delta)$ and introduce the notation

$$
f_{i k}(t)=\rho_{i k} e^{i \alpha_{i k}} \quad(i, k=1,2, \ldots),
$$

\footnotetext{
${ }^{6}$ This can be seen e.g. by the aid of the three series theorem of Kolmogorov.
} 
where $-\pi<\alpha_{i k} \leq \pi$. Since (2.13) converges, it follows that

$$
\prod_{r=1}^{\infty} \prod_{s=1}^{\infty} \rho_{i_{r} k_{s}^{(r)}}
$$

does also. Hence a well-known argument shows that

$$
\sum_{i=1}^{\infty} \sum_{k=1}^{\infty}\left(1-\rho_{i k}\right)<\infty .
$$

Now consider the double series

$$
\sum_{i=1}^{\infty} \sum_{k=1}^{\infty} \alpha_{i k}
$$

and prove that it converges absolutely. Let us suppose that it does not hold. In this case either the sum of the positive terms or that of the negative terms diverges. We consider the first case, the second one can be treated similarly. Then there is a countable number of rows of the matrix $\left(\alpha_{i k}\right)$ which contain the positive elements. If $j_{r}, l_{s}^{(r)}(s=1,2, \ldots ; r=$ $1,2, \ldots)$ denote the positions of the positive elements, then the convergence of the infinite product

$$
\prod_{s} f_{j_{r} l_{s}^{(r)}} \quad(r=1,2, \ldots)
$$

implies that

$$
\alpha_{j_{r}}=\sum_{s} \alpha_{j_{r} l_{s}^{(r)}}<\infty
$$

We can distinguish two cases accordingly as $\alpha_{j_{r}} \leq \pi$ for every large $r$ or $\alpha_{j_{r}}>\pi$ for an infinite number of the $r$ 's. In the first case the convergence of the infinite product

$$
\prod_{n=1}^{\infty} \prod_{s, i, r^{\prime}}
$$

implies

$$
\sum_{r=1}^{\infty} \alpha_{j_{r}}<\infty
$$

what is a contradiction. Considering the second case, let $i_{1}, i_{2}, \ldots$ denote a subsequence of the sequence $j_{1}, j_{2}, \ldots$ for which $\alpha_{i_{r}} \geq \pi(r=1,2, \ldots)$. Then in the $i_{r}$-th row of the matrix there is a finite or infinite number of positions, whose subscripts will be denoted by $k_{1}^{(r)}, k_{2}^{(r)}, \ldots$, such that

$$
\frac{\pi}{2} \leq \sum_{s} \alpha_{i_{r} k_{s}^{(r)}} \leq \pi
$$

This is, however, also a contradiction, since the infinite product

$$
\prod_{r=1}^{\infty} \prod_{s} e^{i \alpha}{ }_{r k_{s}}^{(r)}
$$


converges. Thus

$$
\sum_{i=1}^{\infty} \sum_{k=1}^{\infty}\left|\alpha_{i k}\right|<\infty
$$

In view of the inequality

$$
\left|1-f_{i k}(t)\right| \leq 1-\rho_{i k}+\left|\alpha_{i k}\right|
$$

we obtain

$$
\sum_{i=1}^{\infty} \sum_{k=1}^{\infty}\left|1-f_{i k}(t)\right|<\infty \quad \text { if } \quad|t| \leq \delta,
$$

hence our Lemma follows (cf. [5], p. 115, Theorem 2.7 and p. 118, Corollary 1).

Applying the Lemma for the random variables $\xi_{i k}=\eta\left(C_{i k}\right)$, we conclude that the series

$$
\sum_{k=1}^{\infty} \sum_{N=1}^{\infty} \eta\left(C_{N k}\right)
$$

converges with probability 1 regardless of the order of summation. By Theorem 1.5 we have

$$
\eta\left(B_{k}\right)=\sum_{N=0}^{\infty} \eta\left(C_{N k}\right)
$$

thus the series (2.8) converges with probability 1 regardless of the order of summation.

Let $\eta^{*}(A)(A \in \mathcal{S})$ denote the extended set function of the set function $\eta(A)(A \in \mathcal{R})$. If $E_{N}$ is the following set:

$$
E_{N}=\{h: h \in B,-N \leq \varphi(h)<N\} \quad \text { where } \quad B \in \mathcal{S},
$$

then by Theorem 1.5

$$
\lim _{N \rightarrow \infty} \int_{E_{N}} \varphi(h) \xi(\mathrm{d} A)=\int_{B} \varphi(h) \xi(\mathrm{d} A)
$$

Now, since $\eta^{*}$ and $\eta$ coincide on $\mathcal{R}$ and $\eta$ is the indefinite integral (2.4), we have

$$
\eta^{*}(B)=\lim _{N \rightarrow \infty} \eta^{*}\left(E_{N}\right)=\lim _{N \rightarrow \infty} \eta\left(E_{N}\right)=\lim _{N \rightarrow \infty} \int_{E_{N}} \varphi(h) \xi(\mathrm{d} A) .
$$

On the basis of (2.16) and (2.17) we may write

$$
\eta^{*}(B)=\int_{B} \varphi(h) \xi(\mathrm{d} A) .
$$

Since the extension process leads to a completely additive stochastic set function, this property of $\eta^{*}$ implies the assertion of our theorem. 
REMARK. Let $\varphi(h)$ be a measurable function taking on a countable number of different values on the set $A \in \mathcal{S}$, i.e. there are measurable disjoint sets $A_{1}, A_{2}, \ldots$ of the $\sigma$-ring AS such that $A=\sum_{k=1}^{\infty} A_{k}$ and $\varphi(h)=\varphi_{k}$ if $h \in A_{k}(k=1,2, \ldots)$. If $\varphi(h)$ is integrable over the sets of the type $A_{i_{1}}+A_{i_{2}}+\cdots$ where $i_{1}, i_{2} \ldots$ is an arbitrary sequence of natural numbers, then (it is integrable over $A$ and)

$$
\int_{A} \varphi(h) \xi(\mathrm{d} A)=\sum_{k=1}^{\infty} \varphi_{k} \xi\left(A_{k}\right),
$$

where the series on the right-hand side converges with probability 1 regardless of the order of summation.

Proof. Consider the $\sigma$-algebra of the sets $A_{i_{1}}+A_{i_{2}}+\cdots$ and denote it by $\overline{\mathcal{S}}$. By condition $\varphi(h)$ is $u$-integrable over the set $A$ with respect to $\overline{\mathcal{S}}$ and the completely additive set function $\xi$. Since $\varphi(h)$ is integrable over $A=A_{1}+A_{2}+\cdots$ with respect to $\mathcal{S}$ and $\xi$, it is integrable with respect to $\overline{\mathcal{S}}$ and $\xi$ too. Applying Theorem 2.4 for $\overline{\mathcal{S}}$ instead of $\mathcal{S}$, the assertion follows.

\section{$\S 3$. Further properties of the integral}

Two properties of the classical Lebesgue-Radon integral cannot be formulated in case of the integral introduced by Definition 3. They will, however, hold in case of the $u$-integral. The first of these properties is that if $\varphi(h)$ and $\psi(h)$ are two measurable functions such that $|\varphi(H)| \leq \psi(h)$, moreover $\psi(h)$ is integrable with respect to some finite measure over a measurable set $A$, then the same holds for $\varphi(h)$.

An example for this is the following: Let $H$ be the set of the natural numbers and $\mathcal{S}$ the $\sigma$-algebra of all subsets of $H$. We define the set function $\xi(A)$ as follows:

$$
\xi(A)=\sum_{h \in A}(-1)^{h+1} \frac{1}{h^{2}} .
$$

It is easy to see that the function

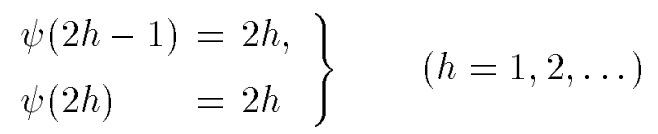

is integrable over $H$, but the same is not true for

$$
\varphi(h)=h \quad(h=1,2, \ldots)
$$

through $0<\varphi(h) \leq \psi(h)$ for every $h$.

A similar example can be given for the assertion that the integrability of two functions $\varphi_{1}(h)$ and $\varphi_{2}(h)$ over a set $A \in \mathcal{S}$ does not imply the integrability of $\varphi_{1}(h)+\varphi_{2}(h)$ over the same set. 
Let $H$ and $\mathcal{S}$ be the same as before and define the functions $\varphi_{1}(h), \varphi_{2}(h)$ as follows:

$$
\left.\begin{array}{l}
\varphi_{1}(2 h)=(-1)^{h} 2 h, \\
\varphi_{1}(2 h-1)=(-1)^{h} 2 h
\end{array}\right\} \quad(h=1,2, \ldots),
$$

In this case the function $\varphi_{1}(h)+\varphi_{2}(h)$ vanishes on the set of the even numbers and its integral does not exist on the set of the positive odd numbers, since the series defining this integral is not absolutely convergent.

As it will be shown in the sequel, these anomalies do not occur in case of the $u$-integral. We prove two theorems, the first of which refers to the $u$-integrability of the sum of two $u$-integrable functions, while the second one, referring to the existence of the $u$-integral of a majorated function (Theorem 2.8), requires further preparations.

THEOREM 2.5. If the functions $\varphi_{1}(h)$ and $\varphi_{2}(h)$ are $u$-integrable over the set $A \in \mathcal{S}$, then the same holds for $\varphi_{1}(h)+\varphi_{2}(h)$ and

$$
\int_{A}\left(\varphi_{1}(h)+\varphi_{2}(h)\right) \xi(\mathrm{d} A)=\int_{A} \varphi_{1}(h) \xi(\mathrm{d} A)+\int_{A} \varphi_{2}(h) \xi(\mathrm{d} A) .
$$

Proof. Let $\left\{y_{k}^{(n)}\right\}$ be an infinitely fine dividing point double sequence and $\left\{H_{k}^{(n)}\right\}$ the corresponding sequence of subdivisions determined by the function $\varphi_{1}(h)+\varphi_{2}(h)$. Now consider the following series:

$$
\begin{aligned}
& \sum_{k=-\infty}^{\infty}\left(y_{k}^{(n)} \xi\left(A H_{k}^{(n)}\right)-\int_{A H_{k}^{(n)}} \varphi_{1}(h) \xi(\mathrm{d} A)-\int_{A H_{k}^{(n)}} \varphi_{2}(h) \xi(\mathrm{d} A)\right) \\
& \quad=\sum_{k=-\infty}^{\infty} y_{k}^{(n)} \xi\left(A H_{k}^{(n)}\right)-\sum_{k=-\infty}^{\infty} \int_{A H_{k}^{(n)}} \varphi_{1}(h) \xi(\mathrm{d} A)-\sum_{k=-\infty}^{\infty} \int_{A H_{k}^{(n)}} \varphi_{2}(h) \xi(\mathrm{d} A) .
\end{aligned}
$$

The second and third series on the right-hand side converge with probability 1 regardless of the order of summation and their sum equals the integrals over $A$ of $\varphi_{1}(h)$ and $\varphi_{2}(h)$, resp. We do not know yet anything about the convergence of the series on the left-hand side.

Let $\left\{z_{s}^{(m)}\right\}$ be an infinitely fine dividing point double sequence, $\left\{L_{s}^{(m)}\right\}$ and $\left\{M_{s}^{(m)}\right\}$ the corresponding sequences of subdivisions determined by the functions $\varphi_{1}(h)$ and $\varphi_{2}(h)$, resp. We introduce the notation $f_{k}^{(n)}(t)$ for the characteristic function of the $k$-th member 
on the left-hand side of (2.24). Since

$$
\begin{aligned}
& y_{k}^{(n)} \xi\left(A H_{k}^{(n)}\right)-\int_{A H_{k}^{(n)}} \varphi_{1}(h) \xi(\mathrm{d} A)-\int_{A H_{k}^{(n)}} \varphi_{2}(h) \xi(\mathrm{d} A) \\
& (2.25)=\lim _{m \rightarrow \infty} \text { st }\left\{y_{k}^{(n)} \xi\left(A H_{k}^{(n)}\right)-\sum_{s} z_{s}^{(m)} \xi\left(A H_{k}^{(n)} L_{s}^{(m)}\right)-\sum_{r} z_{r}^{(m)} \xi\left(A H_{k}^{(n)} M_{r}^{(m)}\right)\right\} \\
& =\lim _{m \rightarrow \infty} \text { st } \sum_{s, r}\left(y_{k}^{(n)}-z_{s}^{(m)}-z_{r}^{(m)}\right) \xi\left(A H_{k}^{(n)} L_{s}^{(m)} M_{r}^{(m)}\right)
\end{aligned}
$$

and in the last member $\left|y_{k}^{(n)}-z_{s}^{(m)}-z_{r}^{(m)}\right| \leq 2 \delta_{n}$ for large $m$ 's where $\delta_{n}=\sup _{k}\left(y_{k+1}^{(n)}\right.$ $\left.-y_{k}^{(n)}\right)$, it follows that

$$
\begin{aligned}
& \left|1-f_{k}^{(n)}(t)\right|=\left|1-\lim _{m \rightarrow \infty} \prod_{s, r} f\left(t\left(y_{k}^{(n)}-z_{s}^{(m)}-z_{r}^{(m)}\right), A H_{k}^{(n)} L_{s}^{(m)} M_{r}^{(m)}\right)\right| \\
& \quad \leq \lim _{m \rightarrow \infty} \sum_{s, r}\left|1-f\left(t\left(y_{k}^{(n)}-z_{s}^{(m)}-z_{r}^{(m)}\right), A H_{k}^{(n)} L_{s}^{(m)} M_{r}^{(m)}\right)\right| \leq W\left(2 \delta_{n} t, A H_{k}^{(n)}\right) .
\end{aligned}
$$

Hence, by summation, we get

$$
\sum_{k=-\infty}^{\infty}\left|1-f_{k}^{(n)}(t)\right| \leq W\left(2 \delta_{n} t, A\right) .
$$

This shows that the series on the left-hand side, consequently also the first series on the right-hand side of (2.24) converges with probability 1 regardless of the order of summation. Taking the limit $n \rightarrow \infty$ in (2.26) and using Theorem 1.1, it follows that the right-hand side of (2.24) tends stochastically to 0 which implies (2.23).

We can repeat the argument for an arbitrary measurable subset of the set $A$ and thus the assertion follows.

\section{$\S 4$. Two auxiliary theorems}

THEOREM 2.6. A non-negative measurable function $\varphi(h)$ is u-integrable over the set $A \in \mathcal{S}$ if and only if for every dividing point sequence $\left\{y_{k}\right\}$, for which $y_{0}=0$, the relation

$$
\sum_{k=0}^{\infty} W\left(y_{k}, A H_{k}\right)<\infty \quad(t \geq 0)
$$

holds where $\left\{H_{k}\right\}$ is the subdivision corresponding to $\varphi(h)$ and $\left\{y_{k}\right\}$.

Proof. The sufficiency of the condition is a simple consequence of Theorem 1.3. In fact, if $B \in A \mathcal{S}$, then (2.27) implies that for every $t$

$$
\sum_{k=0}^{\infty} W\left(t y_{k}, B H_{k}\right) \leq \sum_{k=0}^{\infty} W\left(t y_{k}, A H_{k}\right)<\infty,
$$


hence

$$
\sum_{k=0}^{\infty} \sup _{|z| \leq t}\left|1-f\left(z y_{k}, B H_{k}\right)\right|<\infty
$$

which implies the convergence in every order of the series

$$
\sum_{k=0}^{\infty} y_{k} \xi\left(B H_{k}\right) \text {. }
$$

The other part of the theorem is less obvious. We prove it in an indirect way and suppose that there exist disjoint sets $B_{k l}\left(l=1, \ldots, l_{k} ; k=0,1,2, \ldots\right)$ such that $B_{k l} \in$ $A H_{k} \mathcal{S}\left(l=1, \ldots, l_{k} ; k=0,1,2, \ldots\right)$ and

$$
\sum_{k=0}^{\infty} \sum_{l=1}^{l_{k}} \sup _{|z| \leq t}\left|1-f\left(z y_{k}, A B_{k l}\right)\right|=\infty .
$$

In view of the inequality

$$
\begin{aligned}
|1-g(t)| & \leq \frac{t^{2}}{2} \int_{|x| \leq \varepsilon} x^{2} \mathrm{~d} G(x)+|t|\left|\int_{|x| \leq \varepsilon} x \mathrm{~d} G(x)\right|+2 \int_{|x|>\varepsilon} \mathrm{d} G(x) \\
& \leq 2\left(t^{2}+\frac{1}{t^{2}}\right)\left(\int_{|x| \leq \varepsilon} x^{2} \mathrm{~d} G(x)+\left|\int_{|x| \leq \varepsilon} x \mathrm{~d} G(x)\right|+\int_{|x|>\varepsilon} \mathrm{d} G(x)\right),
\end{aligned}
$$

valid for every $t$, every $\varepsilon>0$ and every distribution function, the characteristic function of which is $g(t)$, we may write

$$
\begin{gathered}
\sum_{k=0}^{\infty} \sum_{l=1}^{l_{k}}\left(\int_{|x| \leq \varepsilon} x^{2} \mathrm{~d} F\left(\frac{x}{y_{k}}, A B_{k l}\right)\right. \\
\left.+\left|\int_{|x| \leq \varepsilon} x \mathrm{~d} F\left(\frac{x}{y_{k}}, A B_{k l}\right)\right|+\int_{|x|>\varepsilon} \mathrm{d} F\left(\frac{x}{y_{k}}, A B_{k l}\right)\right)=\infty .
\end{gathered}
$$

Hence the three series theorem of Kolmogorov implies that the series of independent random variables

$$
\sum_{k=0}^{\infty} \sum_{l=1}^{l_{k}} y_{k} \xi\left(B_{k l}\right)
$$

does not converge with probability 1 regardless of the order of summation.

This leads to a contradiction. Let $K=\sup _{k}\left(y_{k+1}-y_{k}\right)$ and denote by $f_{k l}(t)$ the characteristic function of the random variable

$$
\int_{B_{k l}} \varphi(h) \xi(\mathrm{d} A)-y_{k} \xi\left(B_{k l}\right)=\int_{B_{k l}}\left(\varphi(h)-y_{k}\right) \xi(\mathrm{d} A) .
$$

By Theorem 1.4

$$
\left|1-f_{k l}(t)\right| \leq W\left(K t, B_{k l}\right)
$$


This implies that

$$
\sum_{k=0}^{\infty} \sum_{l=1}^{l_{k}}\left|1-f_{k l}(t)\right|<\infty
$$

hence the series

$$
\sum_{k=0}^{\infty} \sum_{l=1}^{l_{k}}\left(\int_{B_{k l}} \varphi(h) \xi(\mathrm{d} A)-y_{k} \xi\left(B_{k l}\right)\right)
$$

converges with probability 1 regardless of the order of summation. Since $\varphi(h)$ is integrable over all elements of the $\sigma$-ring $A \mathcal{S}$, by Theorem 2.4 the series

$$
\sum_{k=0}^{\infty} \sum_{l=1}^{l_{k}} \int_{B_{k l}} \varphi(h) \xi(\mathrm{d} A)
$$

has also this property. Thus

$$
\sum_{k=0}^{\infty} \sum_{l=1}^{l_{k}} y_{k} \xi\left(B_{k l}\right)
$$

converges with probability 1 regardless of the order of summation what is a contradiction.

Theorem 2.7. Let $\varphi(h)$ and $\psi(h)$ be two non-negative measurable functions such that $\varphi(h) \leq \psi(h)$ and $\psi(h)$ is u-integrable over the set $A(A \in \mathcal{S})$. If $\left\{y_{k}\right\}\left(y_{0}=0\right)$ is a dividing point sequence, then we have

$$
\sum_{k=0}^{\infty} W\left(y_{k}, A L_{k}\right) \leq \sum_{k=0}^{\infty} W\left(y_{k}, A H_{k}\right),
$$

where $\left\{L_{k}\right\}$ and $\left\{H_{k}\right\}$ are the subdivisions corresponding to the functions $\varphi(h)$ and $\psi(h)$, respectively.

Proof. By definition

$$
\left.\begin{array}{l}
L_{k}=\left\{h: y_{k} \leq \varphi(h)<y_{k+1}\right\}, \\
H_{k}=\left\{h: y_{k} \leq \varphi(h)<y_{k+1}\right\}
\end{array}\right\} \quad(k=0,1,2, \ldots),
$$

hence

$$
\begin{gathered}
\sum_{k=0}^{j} H_{k} \subseteq \sum_{k=0}^{j} L_{k} \quad(j=0,1,2, \ldots), \\
H_{j} L_{k}=0 \quad \text { for } \quad k>j .
\end{gathered}
$$


From these we conclude that

$$
\begin{aligned}
\sum_{j=0}^{\infty} W\left(y_{j}, A H_{j}\right) & =\sum_{j=0}^{\infty} \sum_{k=0}^{\infty} W\left(y_{j}, A H_{j} L_{k}\right) \\
& =\sum_{j=0}^{\infty} \sum_{k=0}^{j} W\left(y_{j}, A H_{j} L_{k}\right)=\sum_{k=0}^{\infty} \sum_{j=k}^{\infty} W\left(y_{j}, A H_{j} L_{k}\right) \\
& \leq \sum_{k=0}^{\infty} \sum_{j=k}^{\infty} W\left(y_{k}, A H_{j} L_{k}\right)=\sum_{k=0}^{\infty} W\left(y_{k}, A \sum_{j=k}^{\infty} H_{j} L_{k}\right)=\sum_{k=0}^{\infty} W\left(y_{k}, A L_{k}\right)
\end{aligned}
$$

what was to be proved.

\section{$\S 5$. The theorem relative to the majorated function and the bounded convergence theorem}

In $\S 3$ of this Chapter we have seen that if a function has an integrable majorant, then this does not imply in general the integrability of the majorated function. For a positive assertion in this direction the same additional assumption is needed which has had a fundamental role in Theorem 2.4. Our result is contained in

THeorem 2.8. Let $\varphi(h)$ and $\psi(h)$ be measurable functions such that $|\varphi(h)| \leq \psi(h)$ and $\psi(h)$ is u-integrable over the set $A \in \mathcal{S}$. Then $\varphi(h)$ is also u-integrable over the set A.

Proof. Let be $B \in A \mathcal{S}$. If $B_{1}$ and $B_{2}$ denote those subsets of $B$ where $\varphi(h) \geq 0$ and $\varphi(h)<0$, resp., then Theorems 2.6 and 2.7 imply the integrability of $\varphi(h)$ over $B_{1}$ and $B_{2}$. Applying Theorem 2.2, the assertion follows.

In accordance with the precedings, the analogy to the bounded convergence theorem contains also more assumptions than simple integrability. It seems, however, that Theorems 2.7 and 2.8 express unexpected good properties of our integral. We have only to remind of our paper [18] where an example is given for a completely additive stochastic set function which cannot be represented as the sum of positive and negative parts (cf. pp. 261-262). A remarkable situation is that though in case of the Radon integral the generalized measure can always be decomposed into a difference of two measures, our Theorems 2.7 and 2.8 need not more assumptions than the corresponding Radon integral theorems.

We shall not consider the integrability term by term of series of functions and other statements, but prove the following theorem from which these can easily be deduced:

TheOREM 2.9. Let $\varphi_{N}(h)(N=1,2, \ldots)$ be a sequence of measurable functions such that $\left|\varphi_{N}(h)\right| \leq \psi(h)$ where $\psi(h)$ is u-integrable over the set $A \in \mathcal{S}$. Suppose furthermore that the limit $\varphi(h)=\lim _{N \rightarrow \infty} \varphi_{N}(h)$ exists. In this case the functions $\varphi(h), \varphi_{N}(h)(N=$ 
$1,2, \ldots)$ are also $u$-integrable over the set $A$ and we have the limit relation

$$
\int_{A} \varphi_{N}(h) \xi(\mathrm{d} A) \Rightarrow \int_{A} \varphi(h) \xi(\mathrm{d} A) \quad \text { if } \quad N \rightarrow \infty
$$

Proof. The assertion regarding the integrability of the functions $\varphi(h), \varphi_{N}(h)(N=$ $1,2, \ldots)$ follows at once from Theorem 2.8. According to Theorem 2.5, we may restrict ourselves to the case when $\varphi(h)=0, \varphi_{N}(h) \geq 0(N=1,2, \ldots)$.

First we consider the case of a sequence majorated by a constant and suppose that $\varphi_{N}(h) \leq M$. Let $A_{N}$ denote the following set:

$$
A_{N}=\left\{h: \varphi_{N}(h) \leq \rho\right\},
$$

where $\rho$ is a positive constant. Denoting by $f_{N}(t)$ the characteristic function of the random variable

$$
\int_{A} \varphi_{N}(h) \xi(\mathrm{d} A)
$$

by Theorem 1.4 we have

$$
\begin{aligned}
\left|1-f_{N}(t)\right| & \leq W\left(\rho t, A_{N}\right)+W\left(M t, A-A_{N}\right) \\
& \leq W(\rho t, A)+W\left(M t, A-A_{N}\right) .
\end{aligned}
$$

Let $t$ be a fixed number and choose first $\rho$ so small that the first term on the right-hand side of (2.39) is less than $\varepsilon / 2$. By Theorem 1.1 this is possible. Next we choose $N$ so large that the second term is also less than $\varepsilon / 2$. The possibility of this is a consequence of the relation

$$
\lim _{N \rightarrow \infty} A_{N}=A
$$

which follows from

$$
\lim _{N \rightarrow \infty} \varphi_{N}(h)=0 .
$$

The preceding argument shows that for every $t$

$$
\lim _{N \rightarrow \infty} f_{N}(t)=1
$$

what was to be proved.

Now, turning to the proof of the general case, let us decompose the function $\varphi_{N}(h)$ as

$$
\varphi_{N}(h)=\varphi_{N}^{(1)}(h)+\varphi_{N}^{(2)}(h)
$$

where

$$
\begin{aligned}
& \varphi_{N}^{(1)}(h)=\left\{\begin{array}{cl}
\varphi_{N}(h) & \text { if } \varphi_{N}(h) \leq M \\
0 & \text { otherwise, }
\end{array}\right. \\
& \varphi_{N}^{(2)}(h)=\varphi_{N}-\varphi_{N}^{(1)}(h)
\end{aligned}
$$

and $M$ is a constant of which we shall dispose later. Denote $f_{N}^{(1)}(t)$ and $f_{N}^{(2)}(t)$ the characteristic functions of the random variables

$$
\int_{A} \varphi_{N}^{(1)}(h) \xi(\mathrm{d} A) \quad \text { and } \quad \int_{A} \varphi_{N}^{(2)}(h) \xi(\mathrm{d} A),
$$


respectively, and consider first the latter.

Let $\left\{y_{k}^{(n)}\right\}$ be an infinitely fine dividing point double sequence for which $\left\{y_{k}^{(n)}\right\} \subseteq$ $\left\{y_{k}^{(n+1)}\right\}$ and denote by $\left\{L_{N k}^{(n)}\right\}$ the sequence of subdivisions determined by $\varphi_{N}^{(2)}(h)$ and $\left\{y_{k}^{(n)}\right\}$. If $f_{N n}^{(2)}(t)$ denotes the characteristic function of the random variable

$$
\sum_{k} y_{k}^{(n)} \xi\left(A L_{N k}^{(n)}\right)
$$

then obviously

$$
f_{N n}^{(2)}(t) \Rightarrow f_{N}^{(2)}(t)
$$

if $n \rightarrow \infty$.

Let furthermore $\left\{H_{k}\right\}$ denote the subdivision corresponding to the dividing point sequence $\left\{y_{k}^{(1)}\right\}=\left\{y_{k}\right\}$ and the function $\psi(h)$, finally denote by $K$ the following number: $K=\sup _{k}\left(y_{k+1}-y_{k}\right)$. If $M \geq 1$, then by applying Theorem 2.6 we get

$$
\begin{aligned}
\left|1-f_{N n}^{(2)}(t)\right| & \leq \sum_{k: y_{k}^{(n)} \geq M} W\left(y_{k}^{(n)} t, A L_{N k}^{(n)}\right) \leq \sum_{k: y_{k}^{(1)} \geq M} W\left(y_{k+1}^{(1)} t, A L_{N k}^{(1)}\right) \\
& \leq \sum_{k: y_{k}^{(1)} \geq M} W\left(y_{k}^{(1)}(K+1) t, A L_{N k}^{(1)}\right) \leq \sum_{k: y_{k}=M} W\left(y_{k}(K+1) t, A H_{k}\right) .
\end{aligned}
$$

Hence

$$
\left|1-f_{N}^{(2)}(t)\right| \leq \sum_{k: y_{k} \geq M} W\left(y_{k}(K+1) t, A H_{k}\right)
$$

Fixing the number $t$, first we choose $M$ so large that the right-hand member of (2.41) is less than $\varepsilon / 2$. The possibility of this is ensured by Theorem 2.6. Next, if $N$ is large enough, then

$$
\left|1-f_{N}^{(1)}(t)\right|<\frac{\varepsilon}{2}
$$

If we keep the notation $f_{N}(t)$ for the characteristic function of the random variable

$$
\int_{A} \varphi_{N}(h) \xi(\mathrm{d} A)
$$

then since the sets of those $h$ 's for which $\varphi_{N}^{(1)}(h) \neq 0$ and $\varphi_{N}^{(2)}(h) \neq 0$, respectively, are disjoint, we have

$$
f_{N}(t)=f_{N}^{(1)}(t) f_{N}^{(2)}(t)
$$

whence

$$
\left|1-f_{N}(t)\right| \leq\left|1-f_{N}^{(1)}(t)\right|+\left|1-f_{N}^{(2)}(t)\right|<\varepsilon
$$

for large N's. Hence our theorem is proved. 


\section{$\S 6$. The case of a non-negative set function}

If the random variables $\xi(A)(A \in \mathcal{S})$ are non-negative, then the sequence (1.2) converges not only stochastically to the limit (1.3), but also with probability 1 . This assertion is a special case of the following theorem which is a strong version of Theorem 2.9:

Theorem 2.10. If for every $B \in \mathcal{S}$ we have $\xi(B) \geq 0$ and $\varphi(h), \varphi_{n}(h)(N=1,2, \ldots)$ are measurable functions such that

$$
\lim _{N \rightarrow \infty} \varphi_{N}(h)=\varphi(h), \quad\left|\varphi_{N}(h)\right| \leq \psi(h) \quad(N=1,2, \ldots)
$$

and $\psi(h)$ is $u$-integrable over the set $A \in \mathcal{S}$, then the same holds for $\varphi(h), \varphi_{N}(h)(N=$ $1,2, \ldots)$ and

$$
\lim _{N \rightarrow \infty} \int_{A} \varphi_{N}(h) \xi(\mathrm{d} A)=\int_{A} \varphi(h) \xi(\mathrm{d} A) .
$$

Proof. It is sufficient to consider the case when $\varphi(h)=0, \varphi_{N}(h) \geq 0(N=1,2, \ldots)$. Let $\varepsilon$ be a positive number and $A_{N}$ the following set:

$$
A_{N}=\left\{h: h \in A, \varphi_{N}(h) \geq \varepsilon\right\} .
$$

Thus we obtain

$$
\begin{aligned}
\int_{A} \varphi_{N}(h) \xi(\mathrm{d} A) & =\int_{A_{N}} \varphi_{N}(h) \xi(\mathrm{d} A)+\int_{A-A_{N}} \varphi_{N}(h) \xi(\mathrm{d} A) \\
& \leq \int_{A_{N}} \psi(h) \xi(\mathrm{d} A)+\varepsilon \xi(A)
\end{aligned}
$$

Using Theorem 2.4 of the present paper and Theorem 4.3 of [18], we conclude

$$
\lim _{N \rightarrow \infty} \int_{A} \varphi_{N}(h) \xi(\mathrm{d} A)=0
$$

Hence our assertion follows.

\section{SOME PROPERTIES OF THE INDEFINITE INTEGRAL}

\section{$\S 1$. Derivation of a completely additive set function}

Let us suppose that the function $\varphi(h)$ is integrable over all sets of $\mathcal{S}$ with respect to the completely additive set function $\xi(A)$ and denote by $\eta$ the indefinite integral:

$$
\eta(A)=\int_{A} \varphi(h) \xi(\mathrm{d} A) \quad(A \in \mathcal{S}) .
$$

First we prove that the function $\varphi(h)$ is uniquely determined by the set functions $\xi$ and $\eta$ except at most on a 0 -set of the set function $\xi$. This is expressed by 
TheOREM 3.1. Let $\varphi_{1}(h)$ and $\varphi_{2}(h)$ be two measurable functions integrable over all sets $A \in \mathcal{S}$ with respect to the set function $\xi$ and suppose that for every $A \in \mathcal{S}$ we have

$$
\int_{A} \varphi_{1}(h) \xi(\mathrm{d} A)=\int_{A} \varphi_{2}(h) \xi(\mathrm{d} A) .
$$

Then there exists a set I such that $H-I$ is a 0 -set relative to the set function $\xi$ and

$$
\varphi_{1}(h)=\varphi_{2}(h) \quad \text { if } \quad h \in I
$$

Proof. Let us consider the function $\psi(h)=\varphi_{1}(h)-\varphi_{2}(h)$. According to (3.2), for every $A \in \mathcal{S}$ we have

$$
\int_{A} \psi(h) \xi(\mathrm{d} A)=0
$$

The fulfilment of this relation obviously does not depend on the concrete representation of the random variables $\xi(A)(A \in \mathcal{S})$, or more precisely, it must hold also for other stochastic set functions defined on the elements of $\mathcal{S}$ whenever the so-called finite-dimensional distributions, i.e. the distributions of the vectors $\left(\xi\left(A_{1}\right), \ldots, \xi\left(A_{n}\right)\right)\left(A_{1} \in \mathcal{S}, \ldots, A_{n} \in \mathcal{S}\right)$ for the different set functions remain unchanged.

Let us imagine the sample space, where the random variables $\xi(A)(A \in \mathcal{S})$ are defined, in two exemplars and denote them by $\Omega_{1}$ and $\Omega_{2}$, respectively. We shall consider the product space

$$
\bar{\Omega}=\Omega_{1} \times \Omega_{2}=\left\{\left(\omega_{1}, \omega_{2}\right)\right\} \quad\left(\omega_{1} \in \Omega_{1}, \omega_{2} \in \Omega_{2}\right)
$$

with the product probability measure. Let

$$
\begin{aligned}
& \xi_{1}(A)=\xi_{1}\left(\left(\omega_{1}, \omega_{2}\right), A\right)=\xi\left(\omega_{1}, A\right), \\
& \xi_{2}(A)=\xi_{2}\left(\left(\omega_{1}, \omega_{2}\right), A\right)=\xi\left(\omega_{2}, A\right) .
\end{aligned}
$$

If $A_{1}, \ldots, A_{m}$ and $B_{1}, \ldots, B_{n}$ are arbitrary systems of sets of $\mathcal{S}$, then the vectors

$$
\left(\xi_{1}\left(A_{1}\right), \ldots, \xi_{1}\left(A_{m}\right)\right), \quad\left(\xi_{2}\left(B_{1}\right), \ldots, \xi_{2}\left(B_{n}\right)\right)
$$

are independent. Hence the set function

$$
\xi_{3}(A)=\xi_{1}(A)-\xi_{2}(A) \quad(A \in \mathcal{S})
$$

is completely additive and every random variable $\xi_{3}(A)(A \in \mathcal{S})$ has a (relative to the point 0$)$ symmetric probability distribution. We know furthermore that

$$
\int_{A} \psi(h) \xi_{3}(\mathrm{~d} A)=\int_{A} \psi(h) \xi_{1}(\mathrm{~d} A)-\int_{A} \psi(h) \xi_{2}(\mathrm{~d} A)=0-0=0
$$

If $A_{N}$ is the set of those $h$ 's for which

$$
\frac{1}{N} \leq|\psi(h)| \leq N \quad(N \geq 1),
$$


then obviously, $A_{N} \subseteq A_{N+1}(N=1,2, \ldots)$.

Let $\left\{y_{k}^{(n)}\right\}$ be an infinitely fine dividing point double sequence and $\left\{H_{k}^{(n)}\right\}$ the sequence of subdivisions determined by $\psi(h)$ and $\left\{y_{k}^{(n)}\right\}$. For a fixed $N$ we have

$$
\sum_{k} y_{k}^{(n)} \xi_{3}\left(A_{N} H_{k}^{(n)}\right) \Rightarrow 0
$$

if $n \rightarrow \infty$. Thus the sum in (3.8) satisfies the weak law of large numbers (cf. [7], $\S 22$, Definition 3), hence (cf. [7], $\S 22$, Remark to the Theorem)

$$
\lim _{n \rightarrow \infty} \sum_{k} M\left[\frac{\left(y_{k}^{(n)} \xi_{3}\left(A_{N} H_{k}^{(n)}\right)\right)^{2}}{1+\left(y_{k}^{(n)} \xi_{3}\left(A_{N} H_{k}^{(n)}\right)\right)^{2}}\right]=0 .
$$

Taking into account the definition of the set $A_{N}$, it follows that

$$
\begin{aligned}
0 & =\lim _{n \rightarrow \infty} \sum_{k} M\left[\frac{\left(y_{k}^{(n)} \xi_{3}\left(A_{N} H_{k}^{(n)}\right)\right)^{2}}{1+\left(y_{k}^{(n)} \xi_{3}\left(A_{N} H_{k}^{(n)}\right)\right)^{2}}\right] \\
& \geq \frac{1}{N^{4}} \lim _{n \rightarrow \infty} \sum_{k} M\left[\frac{\left(\xi_{3}\left(A_{N} H_{k}^{(n)}\right)\right)^{2}}{1+\left(\xi_{3}\left(A_{N} H_{k}^{(n)}\right)\right)^{2}}\right] .
\end{aligned}
$$

Applying again the necessary and sufficient condition of the weak law of large numbers we get

$$
\sum_{k} \xi_{3}\left(A_{N} H_{k}^{(n)}\right) \Rightarrow 0
$$

if $n \rightarrow \infty$. But for every $n$ (3.11) equals $\xi_{3}\left(A_{N}\right)$, hence $\xi_{3}\left(A_{N}\right)=0$.

Let $L$ denote the set where $\psi(h) \neq 0$. It follows from the precedings that

$$
\xi_{3}(L)=\lim _{N \rightarrow \infty} \xi_{3}\left(A_{N}\right) .
$$

Now suppose that $B \in L \mathcal{S}$. Since by (3.12)

$$
\xi_{3}(B)+\xi_{3}(L-B)=0
$$

further $\xi_{3}(B)$ and $\xi_{3}(L-B)$ are independent and depend on symmetric distributions (with respect to the point 0 ), we obtain that $\xi_{3}(B)=0$. On the other hand, $\xi_{1}(B)$ and $\xi_{2}(B)$ are also independent and $\xi_{3}(B)=\xi_{1}(B)-\xi_{2}(B)$, hence $\xi_{1}(B)=$ const.

Now we return to our original set function $\xi$. As $\xi(B)$ and $\xi_{1}(B)$ depend on the same probability distribution, we conclude that there is a completely additive number-valued set function $\mu$ defined on the $\sigma$-algebra $L \mathcal{S}$ such that

$$
\xi(B)=\mu(B) \quad \text { for } \quad B \in L \mathcal{S} .
$$


Since

$$
\int_{A} \psi(h) \mu(\mathrm{d} A)=\int_{A} \psi(h) \xi(\mathrm{d} A)=0 \quad(A \in L \mathcal{S})
$$

the Radon-Nikodym theorem implies the existence of a set $I \in L \mathcal{S}$ such that $L-I$ is a 0 -set with regard to $\mu$ (or what is the same, to $\xi$ ) and

$$
\psi(h)=0 \quad \text { if } \quad h \in I .
$$

This set $I$ fulfils the requirements of our theorem.

We shall call the function $\varphi(h)$ the derivative of the completely additive set function $\eta$ relative to the completely additive set function $\xi$ and denote it by

$$
\varphi(h)=\frac{\mathrm{d} \eta}{\mathrm{d} \xi}
$$

We may call also the set function $\eta$ absolutely continuous relative to the set function $\xi$. In the following $\S$ we formulate the chain rule for the derivation of stochastic set functions which is the generalization of the classical Radon-Nikodym theorem.

\section{$\S 2$. The chain rule for the derivation of completely additive stochastic set functions.}

In this section we prove the following

THeOREM 3.2. Let $\xi, \eta, \zeta$ be completely additive stochastic set functions defined on the $\sigma$-ring $\mathcal{S}$. Suppose that there exist measurable functions $\varphi(h)$ and $\psi(h)$ such that for every $A \in \mathcal{S}$

$$
\zeta(A)=\int_{A} \psi(h) \eta(\mathrm{d} A), \quad \eta(A)=\int_{A} \varphi(h) \xi(\mathrm{d} A)
$$

In this case $\varphi(h) \psi(h)$ is integrable over all sets of $\mathcal{S}$ relative to the completely additive set function $\xi$ and

$$
\zeta(A)=\int_{A} \varphi(h) \psi(h) \xi(\mathrm{d} A)
$$

for every $A \in \mathcal{S}$. By other words: the derivative of $\zeta$ relative to $\xi$ exists and

$$
\frac{\mathrm{d} \zeta}{\mathrm{d} \xi}=\frac{\mathrm{d} \zeta}{\mathrm{d} \eta} \frac{\mathrm{d} \eta}{\mathrm{d} \xi}
$$

Proof. Let us suppose first that $\varphi(h) \psi(h)$ is a bounded function. We choose an infinitely fine dividing point double sequence $\left\{y_{k}^{(n)}\right\}$ and denote by $\left\{H_{k}^{(n)}\right\}$ the sequence 
of subdivisions determined by $\psi(h)$ and $\left\{y_{k}^{(n)}\right\}$. Let us define the sequences of functions $\psi_{n}(h), \varphi_{n}(h)$ as follows:

$$
\begin{gathered}
\psi_{n}(h)=y_{k}^{(n)} \quad \text { if } \quad h \in H_{k}^{(n)} \quad(k=0, \pm 1, \pm 2, \ldots ; n=1,2, \ldots), \\
\varphi_{n}(h)=\varphi(h) \psi_{n}(h) \quad(n=1,2, \ldots) .
\end{gathered}
$$

Our assumptions imply

$$
\sum_{k} y_{k}^{(n)} \eta\left(A H_{k}^{(n)}\right) \Rightarrow \zeta(A)
$$

On the other hand,

$$
\begin{aligned}
\sum_{k} y_{k}^{(n)} \eta\left(A H_{k}^{(n)}\right) & =\sum_{k} y_{k}^{(n)} \int_{A H_{k}^{(n)}} \varphi(h) \xi(\mathrm{d} A)=\sum_{k} \int_{A H_{k}^{(n)}} y_{k}^{(n)} \varphi(h) \xi(\mathrm{d} A) \\
& =\sum_{k} \int_{A H_{k}^{(n)}} \varphi(h) \psi_{n}(h) \xi(\mathrm{d} A)=\int_{A} \varphi_{n}(h) \xi(\mathrm{d} A) .
\end{aligned}
$$

Relations (3.21) and (3.22) together imply

$$
\xi(A)=\int_{A} \varphi(h) \psi(h) \xi(\mathrm{d} A) .
$$

Now we return to the general case. First we prove that the function $\varphi(h) \psi(h)$ is integrable over all measurable sets relative to the completely additive set function $\xi$. For this purpose we consider a dividing point sequence $\left\{y_{k}\right\}$ with the corresponding sequence of sets:

$$
H_{k}=\left\{h: y_{k} \leq \varphi(h) \psi(h)<y_{k+1}\right\} \quad(k=0, \pm 1, \pm 2, \ldots) .
$$

According to what has been said above concerning the bounded functions, it follows that

$$
\zeta\left(C H_{k}\right)=\int_{C H_{k}} \varphi(h) \psi(h) \xi(\mathrm{d} A),
$$

where $C$ is an arbitrary but fixed element of the $\sigma$-ring $\mathcal{S}$.

Since $\zeta$ is a completely additive stochastic set function, the series

$$
\sum_{k=-\infty} \zeta\left(C H_{k}\right)
$$

consequently also the series

$$
\sum_{k=-\infty}^{\infty} \int_{C H_{k}} \varphi(h) \psi(h) \xi(\mathrm{d} A)
$$

converges with probability 1 regardless of the order of summation. 
On the other hand, the relation

$$
\varphi(h) \psi(h)=\left(\varphi(h) \psi(h)-y_{k}\right)+y_{k}
$$

implies that

$$
\int_{C H_{k}} \varphi(h) \psi(h) \xi(\mathrm{d} A)=\int_{C H_{k}}\left(\varphi(h) \psi(h)-y_{k}\right) \xi(\mathrm{d} A)+y_{k} \xi\left(C H_{k}\right) .
$$

If $g_{k}(t)$ denotes the characteristic function of the first term on the right-hand side, then by Theorem 1.4 we have

$$
\left|1-g_{k}(t)\right| \leq W\left(\delta t, C H_{k}\right)
$$

where $W$ is the measure defined by (1.4) with respect to the set function $\xi$ and $\delta=$ $\sup _{k}\left(y_{k+1}-y_{k}\right)$. It follows from (3.25) that

$$
\sum_{k=-\infty}^{\infty}\left|1-g_{k}(t)\right| \leq \sum_{k=-\infty}^{\infty} W\left(\delta t, C H_{k}\right)=W(\delta t, C)<\infty .
$$

Thus the series

$$
\sum_{k=-\infty}^{\infty} \int_{C H_{k}}\left(\varphi(h) \psi(h)-y_{k}\right) \xi(\mathrm{d} A)
$$

converges with probability 1 regardless of the order of summation. Taking (3.24) into account we conclude that the series

$$
\sum_{k=-\infty}^{\infty} y_{k} \xi\left(C H_{k}\right)
$$

does also which implies the integrability of $\varphi(h) \psi(h)$ relative to the set function $\xi$ on the set $C$. Since $C$ was arbitrary, our assertion holds.

Now let $A_{N}$ be the following set:

$$
A_{N}=\{h: h \in A,-N \leq \varphi(h) \psi(h)<N\} .
$$

Since the integral of $\varphi(h) \psi(h)$ coincides with the corresponding value of $\zeta$ on every set where $\varphi(h) \psi(h)$ is bounded, it follows that

$$
\zeta\left(A_{N}\right)=\int_{A_{N}} \varphi(h) \psi(h) \xi(\mathrm{d} A) .
$$

If $N \rightarrow \infty$, then the monotonous sequence $A_{N}$ converges to $A$. Moreover, since $\zeta$ and the indefinite integral of the function $\varphi(h) \psi(h)$ are completely additive set functions, (3.26) implies that

$$
\zeta(A)=\lim _{N \rightarrow \infty} \zeta\left(A_{N}\right)=\lim _{N \rightarrow \infty} \int_{A_{N}} \varphi(h) \psi(h) \xi(\mathrm{d} A)=\int_{A} \varphi(h) \psi(h)(\mathrm{d} A) .
$$

Thus Theorem 3.2 is proved. 
Remark. The classical Radon-Nikodym theorem cannot be generalized word for word to our integral. E.g., let $H$ be the interval $[0,1]$ and $\mathcal{S}$ the family of the Lebesgue measurable sets. If $\xi(A)$ and $\eta(A)$ are two completely additive set functions with the characteristic functions

$$
e^{i m(A) t-m(A) \frac{t^{2}}{2}} \quad \text { and } \quad e^{-m(A) \frac{t^{2}}{2}},
$$

respectively, where $m(A)$ is the Lebesgue measure of $A$, then the relation $\xi(A)=0$ implies that $\eta(A)=0$. Nevertheless, the relation

$$
\eta(A)=\int_{A} \varphi(h) \xi(\mathrm{d} A) \quad(A \in \mathcal{S})
$$

can be fulfilled for no function $\varphi(h)$.

In fact, as it is easy to see, the characteristic function of $\eta(A)$ equals

$$
\exp \left\{i t \int_{A} \varphi(h) m(\mathrm{~d} A)-\frac{t^{2}}{2} \int_{A} \varphi^{2}(h) m(\mathrm{~d} A)\right\} \text {. }
$$

For every $A \in \mathcal{S}$ this must be identical with the function of $t$

$$
\exp \left\{-\frac{t^{2}}{2} m(A)\right\}
$$

which is impossible.

\section{SOME THEOREMS CONCERNING THE EXPECTATIONS AND DISPERSIONS}

\section{$\S 1$. The expectations and dispersions of a completely addi- tive set function}

If $\xi(A)$ is an additive set function defined on a ring of sets $\mathcal{R}$ and for every $A \in \mathcal{R}$ the expectation

$$
M(A)=\mathbf{M}(\xi(A))
$$

exists, then $M(A)(A \in \mathcal{R})$ is an additive number-valued set function. The same holds for the variances

$$
D^{2}(A)=\mathbf{D}^{2}(\xi(A))
$$

if they exist. The complete additiveness of the set functions (4.1) and (4.2) holds also if $\xi(A)$ is a completely additive set function. This is expressed by 
TheOREM 4.1. If $\xi(A)$ is a completely additive set function defined on a ring $\mathcal{R}$ for which the expectations $\mathrm{M}(\xi(A))$ or the dispersions $\mathbf{D}(\xi(A))(A \in \mathcal{R})$ exist, then, respectively, the set functions (4.1) and (4.2) are completely additive.

Proof. Let $A_{1}, A_{2}, \ldots$ be a sequence of disjoint sets of $\mathcal{R}$ with $A=\sum_{k=1}^{\infty} A_{k} \in \mathcal{R}$. According to a theorem of Dоoв (cf. [5], p. 339, Theorem 5.2),

$$
\mathbf{M}(\xi(A))=\sum_{k=1}^{\infty} \mathbf{M}\left(\xi\left(A_{k}\right)\right)
$$

whence

$$
M(A)=\sum_{k=1}^{\infty} M\left(A_{k}\right) .
$$

In order to simplify the proof of our assertion relative to the dispersions we suppose that $M(B)=0$ for every $B \in \mathcal{R}$. By the above-mentioned theorem of DooB we have also

$$
\lim _{n \rightarrow \infty} \mathbf{M}\left[\left(\xi(A)-\sum_{k=1}^{n} \xi\left(A_{k}\right)\right)^{2}\right]=0 .
$$

Since

$$
\xi(A)-\sum_{k=1}^{n} \xi\left(A_{k}\right)=\xi\left(B_{n+1}\right),
$$

where $B_{n}=\sum_{k=n}^{\infty} A_{k}$, we have

$$
\lim _{n \rightarrow \infty} D^{2}\left(B_{n+1}\right)=\lim _{n \rightarrow \infty} \mathbf{D}^{2}\left(\xi\left(B_{n+1}\right)\right)=0 .
$$

On the other hand,

$$
D^{2}(A)=\sum_{k=1}^{n} D^{2}\left(A_{k}\right)+D^{2}\left(B_{n+1}\right)
$$

whence the theorem follows.

REMARK. If $\xi(A)(A \in \mathcal{R})$ is a completely additive set function and for every $A \in \mathcal{S}$ the dispersion $D^{2}(A)=\mathrm{D}^{2}(\xi(A))$ exists, further the completely additive number-valued set functions (4.1) and (4.2) can be extended to $\mathcal{S}(\mathcal{R})$, then $\xi(A)$ can also be extended to $\mathcal{S}(\mathcal{R})$ and

$$
\mathbf{D}^{2}\left(\xi^{*}(B)\right)=D^{* 2}(B), \quad \mathbf{M}\left(\xi^{*}(B)\right)=M^{*}(B) \quad(B \in \mathcal{S}(\mathcal{R})),
$$

where $\xi^{*}, D^{* 2}$ and $M^{*}$ denote the extended set functions corresponding to $\xi, D^{2}$ and $M$, respectively.

Proof. According to Theorem 3.11 of [18], the completely additive set function $\xi(A)-$ $M(A)(A \in \mathcal{R})$ can be extended to $\mathcal{S}(\mathcal{R})$. If $\mathfrak{M}$ denotes the family of those sets $B$ for which $\xi^{*}(B)$ has a finite dispersion and (4.6) holds, then from the well-known theorems concerning the convergence of series of independent random variables it follows that $\mathfrak{M}$ is a monotone class of sets (cf. [8], p. 27). Since $\mathcal{R} \subseteq \mathfrak{M}$, we conclude that $\mathfrak{M}=\mathcal{S}(\mathcal{R})$ (cf. [8] p. 27, Theorem B). 


\section{$\S 2$. The expectations and dispersions of the integral (1.3)}

In general, the existence of the expectations $\mathbf{M}(\xi(A))(A \in \mathcal{S})$ (and dispersions $\mathbf{D}^{2}(\xi(A)$ ) $(A \in \mathcal{S})$, resp.) does not imply the existence of the corresponding quantities of the integral (1.3). In order to prove such a theorem we have to make further assumptions. We shall prove two theorems in this direction. In the first one we do not strive to formulate a very general assertion.

Theorem 4.2. Let $\xi(A)(A \in \mathcal{S})$ be a completely additive set function. Suppose that for every $B \in \mathcal{S}$ the expectation $M(B)=\mathrm{M}(\xi(B))$ exists and there is a random variable $\zeta$ such that $\mathrm{M}(|\zeta|)<\infty$ and

$$
\sum_{k=1}^{r}\left|\xi\left(A_{k}\right)\right| \leq \zeta
$$

where $A_{1}, \ldots, A_{r}$ is an arbitrary system of disjoint sets of $\mathcal{S}$. In this case for every bounded and measurable function $\varphi(h)$ the expectations of the random variables

$$
\eta(B)=\int_{B} \varphi(h) \xi(\mathrm{d} A)
$$

exist and

$$
\mathrm{M}(\eta(B))=\int_{B} \varphi(h) M(\mathrm{~d} A)
$$

where the integral is taken in the sense of Radon.

Proof. Let $\left\{y_{k}^{(n)}\right\}$ be an infinitely fine dividing point double sequence and $K$ the upper bound of the values of $|\varphi(h)|$. Consider the series

$$
\eta_{n}=\sum_{k=-\infty}^{\infty} y_{k}^{(n)} \xi\left(B H_{k}^{(n)}\right)
$$

where $\left\{H_{k}^{(n)}\right\}$ is the sequence of subdivisions determined by $\left\{y_{k}^{(n)}\right\}$ and $\varphi(h)$. Since

$$
\eta_{n} \Rightarrow \int_{B} \varphi(h) \xi(\mathrm{d} A) \quad \text { if } \quad n \rightarrow \infty
$$

moreover

$$
\left|\eta_{n}\right| \leq \sum_{k=-\infty}^{\infty}\left|y_{k}^{(n)}\right|\left|\xi\left(B H_{k}^{(n)}\right)\right| \leq K \zeta
$$

the bounded convergence theorem of Lebesgue (cf. [8], §26, Theorem 4) implies that

$$
\lim _{n \rightarrow \infty} \mathbf{M}\left(\eta_{n}\right)=\mathbf{M}(\eta(B)) .
$$

In view of (4.9) we have also

$$
\mathbf{M}\left(\eta_{n}\right)=\sum_{k=-\infty}^{\infty} y_{k}^{(n)} \mathbf{M}\left(\xi\left(B H_{k}^{(n)}\right)\right)=\sum_{k=-\infty}^{\infty} y_{k}^{(n)} M\left(B H_{k}^{(n)}\right) .
$$

Comparing (4.10) and (4.11), the theorem follows. 
Theorem 4.3. Let $\xi(A)(A \in \mathcal{S})$ be a completely additive set function. Suppose that for every $B \in \mathcal{S}$ the dispersion $D^{2}(B)=\mathbf{D}^{2}(\xi(B))$ exists and $\varphi(h)$ is such a measurable function that the integrals

$$
\int_{B} \varphi(h) M(\mathrm{~d} A), \quad \int_{B} \varphi^{2}(h) D^{2}(\mathrm{~d} A)
$$

exist. In this case the integral (4.7), $\mathrm{M}(\eta(B))$ and $\mathbf{D}^{2}(\eta(B))$ also exist, (4.8) holds and

$$
D^{2}(\eta(B))=\int_{B} \varphi^{2}(h) D^{2}(\mathrm{~d} A)
$$

Proof. Let $\left\{y_{k}^{(n)}\right\}$ be an infinitely fine dividing point double sequence and $\left\{H_{k}^{(n)}\right\}$ the sequence of subdivisions determined by $\left\{y_{k}^{(n)}\right\}$ and the function $\varphi(h)$. We show that the sequence

$$
\zeta_{n}=\sum_{k} y_{k}^{(n)} \xi\left(B H_{k}^{(n)}\right)
$$

converges in mean. In fact, if $\left\{z_{k}\right\}$ is the union of the dividing point sequences $\left\{y_{k}^{(n)}\right\}$, $\left\{y_{k}^{(m)}\right\}$ (not indicating the dependence on $n$ and $m$ ) and $\left\{L_{k}\right\}$ is the corresponding sequence of subdivisions, then

$$
\zeta_{n}-\zeta_{m}=\sum_{k} \sum_{j: L_{j} \subseteq H_{k}^{(n)}}\left(y_{k}^{(n)}-z_{j}\right) \xi\left(A L_{j}\right)-\sum_{k} \sum_{j: L_{j} \subseteq H_{k}^{(m)}}\left(y_{k}^{(m)}-z_{j}\right) \xi\left(A L_{j}\right) .
$$

If $\delta_{k j}^{(n)}=y_{k}^{(n)}-z_{j}$, then, since the double sequence $\left\{y_{k}^{(n)}\right\}$ is infinitely fine, it follows

$$
\delta^{(n)}=\sup _{k} \max _{j: L_{j} \subseteq H_{k}^{(n)}}\left|\delta_{k j}^{(n)}\right| \rightarrow 0 \quad \text { if } \quad n \rightarrow \infty .
$$

Formulae (4.13) and (4.14) imply

$$
\begin{aligned}
\mathbf{D}^{2}\left(\zeta_{n}-\zeta_{m}\right) \leq & 2 \sum_{k} \sum_{j: L_{j} \subseteq H_{k}^{(n)}}\left(y_{k}^{(n)}-z_{j}\right)^{2} \mathbf{D}^{2}\left(\xi\left(A L_{j}\right)\right) \\
& +2 \sum_{k} \sum_{j: L_{j} \subseteq H_{k}^{(m)}}\left(y_{k}^{(m)}-z_{j}\right)^{2} \mathbf{D}^{2}\left(\xi\left(A L_{j}\right)\right) \\
\leq & 4\left(\delta^{(n)}\right)^{2} \sum_{j} \mathbf{D}^{2}\left(\xi\left(A L_{j}\right)\right)=4\left(\delta^{(n)}\right)^{2} \mathbf{D}^{2}(\xi(A)) \rightarrow 0 \quad \text { if } \quad m, n \rightarrow \infty
\end{aligned}
$$

and it is easy to see that $\mathrm{M}\left(\zeta_{n}-\zeta_{m}\right) \rightarrow 0$ for $m, n \rightarrow \infty$. We know that

$$
\zeta \Rightarrow \eta(B) \quad \text { if } \quad n \rightarrow \infty
$$


hence

$$
\left.\begin{array}{l}
\mathrm{M}\left(\zeta_{n}\right) \rightarrow \mathrm{M}(\eta(B)), \\
\mathbf{M}\left(\zeta_{n}^{2}\right) \rightarrow \mathbf{M}\left(\eta^{2}(B)\right)
\end{array}\right\} \quad \text { if } \quad n \rightarrow \infty
$$

Using the fact that the random variables $\zeta\left(B H_{k}^{(n)}\right)(k=0, \pm 1, \pm 2, \ldots)$ are independent, we get

$$
\mathbf{D}^{2}\left(\zeta_{n}\right)=\mathbf{M}\left(\zeta_{n}^{2}\right)-\mathbf{M}^{2}\left(\zeta_{n}\right)=\sum_{k}\left(y_{k}^{(n)}\right)^{2} D^{2}\left(B H_{k}^{(n)}\right)
$$

whence (4.11) follows immediately. (4.18) is equivalent to the first line of (4.17).

A similar formula could be deduced for the third central moments. In fact, the third central moment of a sum of independent random variables is equal to the sum of the single third central moments. As this question is not a very significant one, we do not enter into the details.

\section{The Characteristic Functional}

Let $\mathcal{B}$ denote the space of those measurable functions $\varphi(h)(h \in H)$ which are almost everywhere bounded relative to the completely additive set function $\xi(A)(A \in \mathcal{S})$. If we assign to every $\varphi \in \mathcal{B}$ the norm

$$
\|\varphi\|=\underset{h \in H}{\operatorname{vrai} \max } \varphi(h)
$$

then $\mathcal{B}$ becomes a Banach space.

We consider the functional

$$
L(\varphi)=\mathbf{M}\left[\exp i \int_{H} \varphi(h) \xi(\mathrm{d} A)\right]
$$

which will be called the characteristic functional of the completely additive set function $\xi$.

The notion of characteristic functional as a generalization of the Fourier integral was introduced by A. N. Kolmogorov [15] in the year 1935. For probabilistic applications it was introduced by L. LE CAM [3] in 1947 for stochastic processes and in the same year by S. Bochner [1] for random additive set functions. The characteristic functional (5.1) is neither a special case nor a generalization of the notions introduced by the abovementioned authors. If the realizations of $\xi(A)$, i.e. the number-valued set functions $\xi(\omega, A)$ for fixed $\omega$ 's are completely additive, then the functional (5.1) becomes a special case of the characteristic functional of Kolmogorov.

If the functional (5.1) is known, then we know also the probability situations concerning the random variables $\xi(A)(A \in \mathcal{S})$. This is expresses more precisely by 
TheOREM 5.1. The characteristic functional $L(\varphi)$ determines completely the probability measure on the smallest $\sigma$-ring relative to which the random variables $\xi(A)(A \in \mathcal{S})$ are measurable.

Proof. Let $A_{1}, \ldots, A_{n}$ be a system of disjoint sets of $\mathcal{S}$. We show that $L(\varphi)$ determines the joint characteristic function of the random variables $\xi\left(A_{1}\right), \ldots, \xi\left(A_{n}\right)$. Let us define the functions

$$
\varphi_{t_{k}}(h)=\left\{\begin{array}{ll}
t_{k} & \text { if } h \in A_{k} \\
0 & \text { otherwise }
\end{array} \quad(k=1, \ldots, n) .\right.
$$

Obviously,

$$
\mathbf{M}\left[\exp i\left(t_{1} \xi\left(A_{1}\right)+\cdots+t_{n} \xi\left(A_{n}\right)\right)\right]=\mathbf{M}\left[\exp i \int_{H} \varphi_{t_{1}, \ldots, t_{n}}(h) \xi(\mathrm{d} A)\right],
$$

where

$$
\varphi_{t_{1}, \ldots, t_{n}}(h)=\sum_{k=1}^{n} \varphi_{t_{k}}(h) .
$$

If the variables $t_{1}, \ldots, t_{n}$ run over the set of the real numbers, then $L\left(\varphi_{t_{1}, \ldots, t_{n}}(h)\right)$ gives the characteristic function of the random vector $\left(\xi\left(A_{1}\right), \ldots, \xi\left(A_{n}\right)\right)$. These so-called finitedimensional distributions determine the probability measure on the $\sigma$-ring in question.

If for every $\varphi \in \mathcal{B}$ the moment

$$
L_{n}(\varphi)=\mathrm{M}\left[\left(\int_{H} \varphi(h) \xi(\mathrm{d} A)\right)^{n}\right]
$$

exists, then we call the functional (5.4) the $n$-th moment of the completely additive set function $\xi(A)(A \in \mathcal{S})$. By Taylor expansion we get from (5.1)

$$
L(\varphi)=1+i L_{1}(\varphi)+i^{2} \frac{L_{2}(\varphi)}{2 !}+\cdots+i^{n-1} \frac{L_{n-1}(\varphi)}{(n-1) !}+i^{n} \frac{L_{n}^{\prime}(\varphi)}{n !} R_{n}(\varphi),
$$

where

$$
L_{n}^{\prime}(\varphi)=M\left(\left|\int_{H} \varphi(h) \xi(\mathrm{d} A)\right|^{2}\right)
$$

and $R_{n}(\varphi)$ is such a functional that $\left|R_{n}(\varphi)\right| \leq 1$.

Now we describe the characteristic functional of some simple type of stochastic completely additive set functions.

1. Poisson type. In this case

$$
f(t, A)=e^{\lambda(A)\left(e^{i t}-1\right)} \quad(A \in \mathcal{S})
$$

where $\lambda(A)$ is a finite measure on the $\sigma$-ring $\mathcal{S}$. It is easy to see that

$$
L(\varphi)=\exp \left\{\int_{H}\left(e^{i \varphi(h)}-1\right) \lambda(\mathrm{d} A)\right\}
$$


We mention that every measurable function $\varphi(h)$ (bounded and not bounded equally) is integrable with respect to a completely additive set function of Poisson type. This is plausible because essentially it is composed of a set of purely discontinuous measures (which are the realizations) with finite numbers of discontinuity points. In this case the realizations are completely additive set functions but it is not difficult to prove the general assertion.

In fact, the convergence with probability 1 of the series

$$
\sum_{k} \xi\left(A H_{k}^{(n)}\right)
$$

implies that for a fixed $n \xi\left(A H_{k}^{(n)}\right) \neq 0$ for at most a finite number of $k$ 's, hence

$$
\sum_{k} y_{k}^{(n)} \xi\left(A H_{k}^{(n)}\right)
$$

also converges with probability 1 . Here $\left\{y_{k}^{(n)}\right\}$ and $\left\{H_{k}^{(n)}\right\}$ have the usual meaning.

2. The compound Poisson type. In this case

$$
f(t, A)=\exp \left\{\sum_{k=1}^{\infty} C_{k}(A)\left(e^{i \lambda_{k} t}-1\right)\right\} \quad(A \in \mathcal{S}),
$$

where $\lambda_{1}, \lambda_{2}, \ldots$ is an additive semi-group of real numbers, further $C_{k}(A)(k=1,2, \ldots)$ and

$$
C(A)=\sum_{k=1}^{\infty} C_{k}(A)
$$

are finite measures on the $\sigma$-ring $\mathcal{S}$.

The characteristic functional has the form

$$
L(\varphi)=\exp \left\{\sum_{k=1}^{\infty} \int_{H}\left(e^{i \lambda_{k} \varphi(h)}-1\right) C_{k}(\mathrm{~d} A)\right\} .
$$

Such as in 1 we can prove that every measurable function $\varphi(h)$ is integrable with respect to a compound stochastic set function too.

3. The Laplace-Gauss type. In this case

$$
f(t, A)=e^{i t M(A)-\frac{D^{2}(A)}{2} t^{2}} \quad(A \in \mathcal{S}),
$$

where $M(A)(A \in \mathcal{S})$ is a number-valued completely additive set function and $D^{2}(A)$ $(A \in \mathcal{S})$ is a finite measure.

The characteristic functional has the form

$$
L(\varphi)=\exp \left\{i t \int_{H} \varphi(h) M(\mathrm{~d} A)-\frac{t^{2}}{2} \int_{H} \varphi^{2}(h) D^{2}(\mathrm{~d} A)\right\}
$$


where the integrals on the right-hand side in the exponent are Radon and Lebesgue integrals, respectively. Formula (5.13) can be deduced immediately from Theorem 4.3 too.

4. The Cauchy type. A completely additive set function $\xi(A)(A \in \mathcal{S})$ will be called of Cauchy type if

$$
f(t, A)=e^{i C_{1}(A) t-C_{2}(A)|t|} \quad(A \in \mathcal{S}),
$$

where $C_{1}(A)(A \in \mathcal{S})$ is a number-valued completely additive set function and $C_{2}(A)$ $(A \in \mathcal{S})$ is a finite measure.

The characteristic functional has the following form:

$$
L(\varphi)=\exp \left\{i \int_{H} \varphi(h) C_{1}(\mathrm{~d} A)-\int_{H}|\varphi(h)| C_{2}(\mathrm{~d} A)\right\}
$$

where the integrals on the right-hand side in the exponent are Radon and Lebesgue integrals, respectively.

It would be possible to define the functional $L(\varphi)$ in a much more extensive space of the functions $\varphi$, e.g. in the space of all functions which are integrable over the set $H$. This latter space is, however, not a Banach space. On the other hand, Theorem 5.1 shows that our space $\mathcal{B}$ is extensive enough to characterize the probabilistic situation concerning a completely additive stochastic set function.

\section{References}

[1] S. Bochner, Stochastic processes, Annals of Math., 48 (1947), pp. 1014-1061.

[2] S. Bochner, Harmonic analysis and the theory of probability (Berkeley - Los Angeles, 1955).

[3] L. LE CAM, Un instrument d'étude des fonctions aléatoires: la fonctionnelle caractéristique, Comptes Rendus Acad. Sci. Paris, 224 (1947), pp. 710-711.

[4] H. Cramér, A contribution to the theory of stochastic processes, Proc. Sec. Berkeley Symp., (1951), pp. 329-340.

[5] J. L. Doob, Stochastic processes (New York-London, 1953).

[6] V. Fabian, Measures the values of which are classes of equivalent measurable functions, Cehoslovackij Mat. Žurnal, 7 (1957), pp. 191-234.

[7] B. V. Gnedenko i A. N. Kolmogorov, Prevedel'nye raspredeleniâ dlâ summ nezavisimyh slučajnyh veličin (Moszkva-Leningrad, 1949).

[8] P. Halmos, Measure theory (New York, 1950).

[9] K. Іто̂, Stochastic processes, Jap. Journal of Math., 18 (1942), pp. 261-301.

[10] K. Iтô, Stochastic integral, Proc. Imp. Acad. Tokyo, 20 (1944), pp. 519-524.

[11] K. Iтô, Multiple Wiener integral, Journal Math. Soc. Japan, 3 (1951), pp. 157-169.

[12] K. Ітô, On stochastic differential equations, Memoirs Amer. Soc., No. 4 (1951).

[13] A. N. Kolmogorov, Kurven im Hilbertschen Raum, die gegenüber einer einparametrigen Gruppe von Bewegungen invariant sind, Doklady Akad. Nauk S.S.S.R., 26 (1940), pp. 6-9. 
[14] A. N. Kolmogorov, Wienersche Spiralen und einige andere interessante Kurven im Hilbertschen Raum, Doklady Akad. Nauk S.S.S.R., 26 (1940), pp. 115-118.

[15] A. N. Kolmogorov, La transformation de Laplace dans les espaces linéaires, Comptes Rendus Acad. Sci. Paris, 200 (1935), pp. 1717-1718.

[16] P. LÉvi, Théorie de l'addition des variables aléatoires (Paris, 1937).

[17] A. Овukнov, On the energy distribution in the spectrum of a turbulent flow, Doklady Akad. Nauk. S.S.S.R., 32 (1941), pp. 19-21.

[18] A. Prékopa, On stochastic set functions. I, Acta Acad. Sci. Hung., 7 (1956), pp. 215263.

[19] J. Radon, Theorie und Anwendung der absolut-additiven Mengenfunktionen, Sitzber. Akad. Wiss. Math. Naturwiss., 122 (1913), pp. 1295-1438.

[20] F. Riesz et B. Sz.-NAgy, Leçons d'analyse fonctionnelle (Budapest, 1952).

[21] N. Wiener, Differential space, Journal Math. Phys. Math. Inst. Tech., 2 (1923), pp. 131-174. 